ESAIM: PROCEEDINGS, August 2008, Vol. 24, p. 77-96

C. Dobrzynski, P. Frey, Ph. Pebay, Editors

\title{
COMPUTABLE A POSTERIORI ERROR ESTIMATES IN THE FINITE ELEMENT METHOD BASED ON ITS LOCAL CONSERVATIVITY: IMPROVEMENTS USING LOCAL MINIMIZATION*,**
}

\author{
Ibrahim Cheddadi ${ }^{1}$, Radek FuČíK ${ }^{2}$, Mariana I. Prieto ${ }^{3}$ And Martin Vohralík ${ }^{4}$
}

\begin{abstract}
We investigate in this paper improvements of the a posteriori error estimates in the finite element method discretization of the Poisson equation, introduced in [M. Vohralík, A posteriori error estimation in the conforming finite element method based on its local conservativity and using local minimization, C. R. Math. Acad. Sci. Paris 346 (2008), 687-690] and [M. Vohralík, Guaranteed and fully robust a posteriori error estimates for conforming discretizations of diffusion problems with discontinuous coefficients, submitted]. The estimates presented in these references are guaranteed in the sense that they feature no undetermined constants and fully computable but numerical experiments show that the effectivity index, i.e., the ratio of the estimated and actual error, does not approach the optimal value of one but rather a slightly bigger value. We identify in this paper the reason for this and introduce a possible remedy, which consists in performing a local minimization of the values of the estimators over patches of simplicial submesh elements. We then present a set of numerical experiments showing the improvements achieved and compare our estimators, both theoretically and numerically, with the classical residual ones.
\end{abstract}

Résumé. Estimations d'erreur a posteriori calculables pour la méthode des éléments finis basées sur la conservativité locale de cette méthode : améliorations employant une minimisation locale. Nous étudions dans cet article des améliorations des estimations a posteriori pour la discrétisation de l'équation de Poisson par la méthode des éléments finis, introduites dans [M. Vohralík, A posteriori error estimation in the conforming finite element method based on its local conservativity and using local minimization, C. R. Math. Acad. Sci. Paris 346 (2008), 687-690] et [M. Vohralík, Guaranteed and fully robust a posteriori error estimates for conforming discretizations of diffusion problems with discontinuous coefficients, soumis]. Les estimations présentées dans ces références sont garanties au sens où elles ne comportent pas de constantes indéterminées et sont entièrement calculables, mais des essais numériques montrent que l'indice d'efficacité, donné par le rapport entre l'erreur estimée et l'erreur exacte, n'approche pas la valeur optimale de un mais une valeur légèrement plus élevée. Dans cet article, nous en identifions la raison et nous introduisons une correction basée sur une minimisation locale des valeurs des estimateurs sur des ensembles de triangles ou de tétraèdres d'un sous-maillage. Nous présentons ensuite une série d'essais numériques et comparons, tant du point de vue théorique que numérique, nos estimations avec les estimations classiques par résidu.

\footnotetext{
* The second author has been partly supported by the project "Applied Mathematics in Technical and Physical Sciences" MSM 6840770010 of the Ministry of Education of the Czech Republic.

** The last author has been partly supported by the GdR MoMaS project "Numerical Simulations and Mathematical Modeling of Underground Nuclear Waste Disposal", PACEN/CNRS, ANDRA, BRGM, CEA, EdF, IRSN, France.

1 Univ. Grenoble and CNRS, Laboratoire Jean Kuntzmann, 51 rue des Mathématiques, 38400 Saint Martin d'Hères \& INRIA Grenoble-Rhône-Alpes, Inovallée, 655 avenue de l'Europe, Montbonnot 38334 Saint Ismier Cedex, France; e-mail: ibrahim. cheddadi@imag.fr

2 Department of Mathematics, Faculty of Nuclear Sciences and Physical Engineering, Czech Technical University in Prague, Trojanova 13, 12000 Prague, Czech Republic; e-mail: fucik@fjfi.cvut.cz

3 Departamento de Matemática, Facultad de Ciencias Exactas y Naturales, Universidad de Buenos Aires, Intendente Güiraldes 2160, Ciudad Universitaria, C1428EGA, Argentina; e-mail: mprieto@dm.uba.ar

4 UPMC Univ. Paris 06, UMR 7598, Laboratoire Jacques-Louis Lions, 75005, Paris, France \& CNRS, UMR 7598, Laboratoire

Jacques-Louis Lions, 75005, Paris, France; e-mail: vohralik@ann.jussieu.fr

(c) EDP Sciences, SMAI 2008
} 


\section{INTRODUCTION}

In the last decades, a vast amount of literature was dedicated to a posteriori error estimation for the continuous piecewise linear finite element approximation of the model diffusion problem

$$
\begin{aligned}
-\Delta p=f & \text { in } \Omega \\
p=0 & \text { on } \partial \Omega
\end{aligned}
$$

where $\Omega \subset \mathbb{R}^{d}, d=2,3$, is a polygonal (polyhedral) domain and $f$ is a source term. Several types of estimators have been developed, such as the averaging ones, cf. Zienkiewicz and Zhu [25], the residual ones, cf. Verfürth [19], or the equilibrated residual ones, cf. Ainsworth and Oden [1]. Many useful properties have been proven about the majority of these estimators, like, e.g., the local efficiency. This property means that each element estimator represents a lower bound for the actual error in the given element or in its neighborhood, up to a multiplicative constant. This property is thus the basis for successful local mesh refinement. In the majority of the cases, however, the estimates are not guaranteed, meaning that a fully computable upper bound on the error is not given. In the averaging approach, for example, the estimate does not give any upper bound on the error, whereas in the residual one, the estimate only gives an upper bound on the error up to an unknown multiplicative constant. Finally, in the equilibrated residual approach, the upper bound is given but it is not computable since it involves a solution of local infinite-dimensional problems. A remedy for this situation in the case of residual estimates was presented by Carstensen and Funken [4], who evaluate the "unknown constants", whence these estimators become guaranteed and fully computable. This result complements some other ones, like, e.g., the works of Ladevèze and Leguillon [13], Destuynder and Métivet [6], Strouboulis et al. [17], Luce and Wohlmuth [14], Vejchodský [18], Repin and Sauter [16], or Korotov [12].

We have recently in [22], [21], and [7] introduced estimators for mixed finite element, finite volume, and discontinuous Galerkin methods which are guaranteed and fully computable, robust with respect to (independent of) the domain and the weak solution, computationally inexpensive and the effectivity index is close to the optimal value of one. Their versions for the finite element method were then presented in [23, 24]. During the CEMRACS 2007 summer research center, we have first implemented these estimators into the FreeFem $++[9]$ code and made a series of numerical experiments. These experiments confirm the theoretical results of [23,24]. In particular, the estimate is guaranteed, fully computable, and robust with respect to the domain and the weak solution, as we show below for a test case where the domain has a reentrant corner and the (known) weak solution possesses a singularity. We have next compared our estimators, for the above model problem and also for a model problem with a regular solution, to the residual ones of Verfürth [19], with the constants evaluated according to Carstensen and Funken [4]. We have concluded here that our estimates lead to much sharper error upper bounds. However, these numerical experiments also showed that the effectivity index still does not approach the optimal value of one.

We have thus consequently focused on the investigation of this issue, identified the overestimation source, and proposed a remedy to this situation. It consists in performing a local minimization of the values of the estimators over patches of simplicial submesh elements sharing a given vertex and leads to a solution of a small linear system for each vertex. As we demonstrate on numerical experiments, this technique helps to considerably decrease the effectivity index. In particular, for the regular test solution, the effectivity index for a sequence of adaptively refined meshes approaches the optimal value of one, which suggests the asymptotic exactness in this case. Also for the solution which possesses a singularity at a reentrant corner, the effectivity index for a sequence of adaptively refined meshes is quite close to one. We note that as the evaluation is local, the overall cost stays a linear function of the number of vertices, which is optimal. Another approach, avoiding any linear system solution, is pursued in [23].

Two other issues, not presented in this paper, were pursued in our project in the CEMRACS 2007 summer research center. First, a generalization of the present results to the reaction-diffusion case, yielding estimates which are guaranteed and moreover robust with respect to the reaction coefficient, is given [5]. Next, all the above-cited results only hold under the condition that the linear systems associated with the numerical scheme 
are solved exactly. This is of course not necessarily true in practice and, moreover, it may be even interesting not to increase the precision of the solution of the linear system if we know that the error is due to insufficient (local) mesh refinement and the discretization (not the algebraic) error. This last point is pursued in [11]. Finally, the estimates in $[7,21,22]$ are derived for convection-diffusion-reaction problems and the present approach in the finite element method can be readily extended to this case. As the overall methodology is quite general, extensions to other types of problems seem possible and will be the subject of a future research.

This paper is organized as follows. In Section 1, we summarize the basic results of [23, 24]. In Section 2, we recall the classical residual estimates of Verfürth [19], with the constants evaluated according to Carstensen and Funken [4]. Next, in Section 3, we present our local minimization strategy and finally, in Section 4, a set of numerical experiments on two model problems is presented.

\section{Guaranteed and fully Computable a posteriori error estimate}

We recall here the basic results of $[23,24]$, giving a guaranteed and fully computable a posteriori error estimate in the finite element method.

\subsection{Notation}

Let $\mathcal{T}_{h}$ denote a conforming simplicial mesh of $\Omega$, i.e., a mesh consisting of triangles $(d=2)$ or tetrahedra ( $d=3$ ) such that $\bar{\Omega}=\bigcup_{K \in \mathcal{T}_{h}} K$ and such that if $K, L \in \mathcal{T}_{h}, K \neq L$, then $K \cap L$ is either an empty set or a common edge, face, or vertex of $K$ and $L$. Next, $\mathcal{V}_{h}\left(\mathcal{V}_{h}^{\text {int }}, \mathcal{V}_{h}^{\text {ext }}\right)$ stand for the set of all (interior, exterior) vertices of $\mathcal{T}_{h}$ and $\mathcal{E}_{h}$ for all the sides (edges if $d=2$, faces if $d=3$ ) of $\mathcal{T}_{h}$. We will also use the notation

$$
\begin{array}{rlrl}
\mathcal{T}_{V} & :=\left\{L \in \mathcal{T}_{h} ; L \cap V \neq \emptyset\right\} & V \in \mathcal{V}_{h}, \\
\mathcal{V}_{K} & :=\left\{V \in \mathcal{V}_{h} ; V \subset K\right\} \quad K \in \mathcal{T}_{h}, \\
\mathcal{E}_{K} & :=\left\{\sigma \in \mathcal{E}_{h} ; \sigma \subset K\right\} \quad K \in \mathcal{T}_{h} .
\end{array}
$$

In addition to $\mathcal{T}_{h}$, we shall here consider dual partitions $\mathcal{D}_{h}$ of $\Omega$. A dual volume $D_{V}$ associated with vertex $V$ (we also use the notation $V_{D}$ for the vertex associated with a given dual volume $D$ ) is constructed as follows. When $d=2, D_{V}$ is the polygon whose vertices are the barycenters of all the triangles $K \in \mathcal{T}_{V}$ and the midpoints of all the edges having $V$ as vertex, see Figure 1 . When $d=3, D_{V}$ is a polyhedron and its vertices are in addition the barycentres of all the faces having $V$ as vertex. We use $\mathcal{D}_{h}^{\text {int }}, \mathcal{D}_{h}^{\text {ext }}$ to denote the dual volumes associated with vertices from $\mathcal{V}_{h}^{\text {int }}, \mathcal{V}_{h}^{\text {ext }}$, respectively.

Finally, we will use a second simplicial mesh $\mathcal{S}_{h}$ of $\Omega$, constructed by dividing each $D \in \mathcal{D}_{h}$ into a mesh $\mathcal{S}_{D}$ as indicated in Figure 1 and then taking $\mathcal{S}_{h}=\bigcup_{D \in \mathcal{D}_{h}} \mathcal{S}_{D}$. We will use the notation $\mathcal{G}_{h}$ for all sides of $\mathcal{S}_{h}$ and $\mathcal{G}_{h}^{\text {int }}\left(\mathcal{G}_{h}^{\text {ext }}\right)$ for all interior (exterior) sides of $\mathcal{S}_{h}$. Next, $\mathbf{n}$ denotes an exterior normal vector, whereas $\mathbf{n}_{\sigma}$ stands for a normal vector of a side $\sigma$ whose orientation is chosen arbitrarily but fixed for interior sides and coinciding with the exterior normal of $\Omega$ for exterior sides. Finally, the jump operator $\llbracket \cdot \rrbracket$ is defined by

$$
\llbracket \varphi \rrbracket:=\left.\left(\left.\varphi\right|_{K}\right)\right|_{\sigma}-\left.\left(\left.\varphi\right|_{L}\right)\right|_{\sigma}
$$

for $\sigma \in \mathcal{G}_{h}^{\text {int }}$ shared by $K, L \in \mathcal{S}_{h}$ such that $\mathbf{n}_{\sigma}$ points from $K$ to $L$. We put $\llbracket \varphi \rrbracket_{\sigma}=0$ for any $\sigma \in \mathcal{G}_{h}^{\text {ext }}$. Similarly, the average operator $\{\{\cdot\}$ is defined by

$$
\left\{\{\varphi\}:=\left.\frac{1}{2}\left(\left.\varphi\right|_{K}\right)\right|_{\sigma}+\left.\frac{1}{2}\left(\left.\varphi\right|_{L}\right)\right|_{\sigma}\right.
$$

for $\sigma \in \mathcal{G}_{h}^{\text {int }}$ shared by $K, L \in \mathcal{S}_{h}$, whereas for $\sigma \in \mathcal{G}_{h}^{\text {ext }},\left\{\{\varphi\}:=\left.\varphi\right|_{\sigma}\right.$. Analogous notation will be used on $\mathcal{E}_{h}$.

Concerning functional notation, we denote by $(\cdot, \cdot)_{S}$ the $L^{2}$-scalar product on $S$ and by $\|\cdot\|_{S}$ the associated norm; when $S=\Omega$, the index is dropped off. We denote by $|S|$ the Lebesgue measure of $S$, by $|\sigma|$ the $(d-1)$ dimensional Lebesgue measure of $\sigma \subset \mathbb{R}^{d-1}$, and in particular by $|\mathbf{s}|$ the length of a segment $\mathbf{s} ; h_{S}$ then stands 


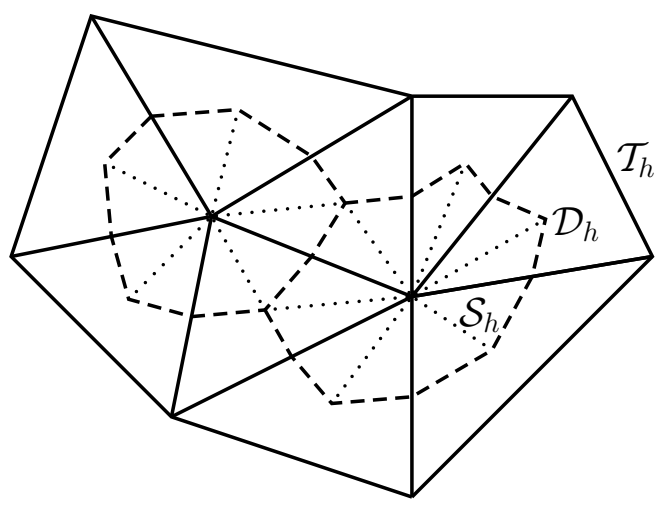

Figure 1. Original simplicial mesh $\mathcal{T}_{h}$, the associated dual mesh $\mathcal{D}_{h}$, and the fine simplicial mesh $\mathcal{S}_{h}$

for the diameter of $S$. Next, $H^{1}(S)$ is the Sobolev space of functions with square-integrable weak derivatives and $H_{0}^{1}(S)$ is its subspace of functions with traces vanishing on $\partial S$. Finally, $\mathbf{H}(\operatorname{div}, S)$ is the space of functions with square-integrable weak divergences, $\mathbf{H}(\operatorname{div}, S)=\left\{\mathbf{v} \in \mathbf{L}^{2}(S) ; \nabla \cdot \mathbf{v} \in L^{2}(S)\right\}$.

\subsection{Continuous and discrete problems}

For the problem (1a)-(1b), we define a bilinear form $\mathcal{B}$ by

$$
\mathcal{B}(p, \varphi):=(\nabla p, \nabla \varphi)
$$

where $p, \varphi \in H_{0}^{1}(\Omega)$, and the associated energy norm by

$$
\|\varphi\|^{2}:=\mathcal{B}(\varphi, \varphi) .
$$

The variational formulation is then to find $p \in H_{0}^{1}(\Omega)$ such that

$$
\mathcal{B}(p, \varphi)=(f, \varphi) \quad \forall \varphi \in H_{0}^{1}(\Omega)
$$

and its finite element approximation consists in finding $p_{h} \in X_{h}^{0}$ such that

$$
\mathcal{B}\left(p_{h}, \varphi_{h}\right)=\left(f, \varphi_{h}\right) \quad \forall \varphi_{h} \in X_{h}^{0},
$$

where

$$
X_{h}^{0}:=\left\{\varphi_{h} \in H_{0}^{1}(\Omega) ;\left.\varphi_{h}\right|_{K} \in \mathbb{P}_{1}(K) \quad \forall K \in \mathcal{T}_{h}\right\}
$$

with $\mathbb{P}_{1}(K)$ the space of linear polynomials on $K \in \mathcal{T}_{h}$.

\subsection{A local conservativity property of the finite element method}

Although this is still not commonly known, the finite element method is locally conservative; not on the primal mesh $\mathcal{T}_{h}$ but on the dual mesh $\mathcal{D}_{h}$, cf., e.g., Bank and Rose [2], Eymard et al. [8, Section III.12], Hughes et al. [10], or [23]. Our a posteriori error estimates are based on this property.

Let $\mathbf{R T N}\left(\mathcal{S}_{h}\right) \subset \mathbf{H}(\operatorname{div}, \Omega)$ be the lowest-order Raviart-Thomas-Nédélec space over the fine simplicial mesh $\mathcal{S}_{h}$; this is a space of vector functions $\mathbf{v}$ having on each $K \in \mathcal{S}_{h}$ the form $\left(a_{K}+d_{K} x, b_{K}+d_{K} y\right)^{t}$ if $d=2$ and $\left(a_{K}+d_{K} x, b_{K}+d_{K} y, c_{K}+d_{K} z\right)^{t}$ if $d=3$, with the normal trace across all $\sigma \in \mathcal{G}_{h}^{\text {int }}$ continuous. Recall that 
$\mathbf{v} \cdot \mathbf{n}_{\sigma}$ is a constant for all $\sigma \in \mathcal{G}_{h}$ and that these side fluxes represent the degrees of freedom of $\mathbf{R T N}\left(\mathcal{S}_{h}\right)$. We now define $\mathbf{t}_{h} \in \mathbf{R T N}\left(\mathcal{S}_{h}\right)$ by

$$
\mathbf{t}_{h} \cdot \mathbf{n}_{\sigma}=-\left\{\left\{\nabla p_{h} \cdot \mathbf{n}_{\sigma}\right\}\right\} \quad \forall \sigma \in \mathcal{G}_{h},
$$

where $p_{h}$ is the finite element approximation given by (5). Note that $\mathbf{t}_{h} \cdot \mathbf{n}_{\sigma}$ is given directly by $-\nabla p_{h} \cdot \mathbf{n}_{\sigma}$ for such $\sigma \in \mathcal{G}_{h}$ where there is no jump in $\nabla p_{h}$, i.e., on all the sides $\sigma \in \mathcal{G}_{h}$ which are in the interior of some $K \in \mathcal{T}_{h}$ or at the boundary of $\Omega$, whereas a simple average of the two normal gradient values is used otherwise. The following important property holds for $\mathbf{t}_{h}$ constructed in this way; we refer to [23, Lemma 5.2] or [24, Lemma 3.1] for the proof.

Lemma 1.1 (Local conservativity of the finite element method on the dual grid $\mathcal{D}_{h}$ ). Let $f$ be piecewise constant on $\mathcal{T}_{h}$ and let $\mathbf{t}_{h}$ by given by (6). Then

$$
\left(\nabla \cdot \mathbf{t}_{h}, 1\right)_{D}=(f, 1)_{D} \quad \forall D \in \mathcal{D}_{h}^{\text {int }}
$$

\subsection{Poincaré and Friedrichs inequalities}

The two following inequalities play a crucial role in our a posteriori error estimates, as well as in the evaluation of the constants in the residual estimates by Carstensen and Funken [4].

Let $D$ be a polygon or a polyhedron. The Poincaré inequality states that

$$
\left\|\varphi-\varphi_{D}\right\|_{D}^{2} \leq C_{\mathrm{P}, D} h_{D}^{2}\|\nabla \varphi\|_{D}^{2} \quad \forall \varphi \in H^{1}(D)
$$

where $\varphi_{D}$ is the mean of $\varphi$ over $D$ given by $\varphi_{D}:=(\varphi, 1)_{D} /|D|$ and where the constant $C_{\mathrm{P}, D}$ can for each convex $D$ be evaluated as $1 / \pi^{2}$, cf. [3,15]. To evaluate $C_{\mathrm{P}, D}$ for nonconvex elements $D$ is more complicated but it still can be done, cf. Carstensen and Funken [4, Section 2] or Eymard et al. [8, Lemma 10.2].

The Friedrichs inequality states that

$$
\|\varphi\|_{D}^{2} \leq C_{\mathrm{F}, D, \partial \Omega} h_{D}^{2}\|\nabla \varphi\|_{D}^{2} \quad \forall \varphi \in H^{1}(D) \text { such that } \varphi=0 \text { on } \partial \Omega \cap \partial D \neq \emptyset .
$$

As long as $\partial \Omega$ is such that there exists a vector $\mathbf{b} \in \mathbb{R}^{d}$ such that for almost all $\mathbf{x} \in D$, the first intersection of $\mathcal{B}_{\mathbf{x}}$ and $\partial D$ lies in $\partial \Omega$, where $\mathcal{B}_{\mathbf{x}}$ is the straight semi-line defined by the origin $\mathbf{x}$ and the vector $\mathbf{b}, C_{\mathrm{F}, D, \partial \Omega}=1$, cf. [20, Remark 5.8]. To evaluate $C_{\mathrm{F}, D, \partial \Omega}$ in the general case is more complicated but it still can be done, cf. Carstensen and Funken [4, Section 3] or [20, Remark 5.9].

\subsection{The a posteriori error estimate}

The following result has been proved in $[23,24]$ :

Theorem 1.2 (Guaranteed and fully computable a posteriori error estimate). Let $p$ be the weak solution of problem (1a)-(1b) given by (4) and let $p_{h} \in H_{0}^{1}(\Omega)$ be arbitrary. Let next $\mathbf{t}_{h} \in \mathbf{H}(\operatorname{div}, \Omega)$ be such that

$$
\left(\nabla \cdot \mathbf{t}_{h}, 1\right)_{D}=(f, 1)_{D} \quad \forall D \in \mathcal{D}_{h}^{\text {int }},
$$

let the diffusive flux estimator $\eta_{\mathrm{DF}, D}$ be given by

$$
\eta_{\mathrm{DF}, D}:=\left\|\nabla p_{h}+\mathbf{t}_{h}\right\|_{D} \quad D \in \mathcal{D}_{h},
$$

and the residual estimator $\eta_{\mathrm{R}, D}$ by

$$
\eta_{\mathrm{R}, D}:=m_{D}\left\|f-\nabla \cdot \mathbf{t}_{h}\right\|_{D} \quad D \in \mathcal{D}_{h},
$$


where

$$
\begin{aligned}
& m_{D}:=C_{\mathrm{P}, D}^{1 / 2} h_{D} \quad D \in \mathcal{D}_{h}^{\text {int }}, \\
& m_{D}:=C_{\mathrm{F}, D, \partial \Omega}^{1 / 2} h_{D} \quad D \in \mathcal{D}_{h}^{\text {ext }},
\end{aligned}
$$

with $C_{\mathrm{P}, D}$ the constant from the Poincaré inequality (8) and $C_{\mathrm{F}, D, \partial \Omega}$ the constant from the Friedrichs inequality (9). Then

$$
\left\|p-p_{h}\right\| \leq\left\{\sum_{D \in \mathcal{D}_{h}}\left(\eta_{\mathrm{R}, D}+\eta_{\mathrm{DF}, D}\right)^{2}\right\}^{1 / 2} .
$$

Note in particular that the above theorem holds true for an arbitrary $\mathbf{t}_{h} \in \mathbf{H}(\operatorname{div}, \Omega)$ satisfying (10), and that (6) thanks to Lemma 1.1 seems as the most natural choice.

\subsection{Efficiency of the a posteriori error estimate}

The local efficiency of the a posteriori error estimate of Theorem 1.2 is given in the following theorem. We refer to [23, Theorem 6.1] for the proof (done in a more general setting).

Theorem 1.3 (Local efficiency of the a posteriori error estimate of Theorem 1.2). Let $f$ be piecewise constant on $\mathcal{T}_{h}$, let $p$ the weak solution of problem (1a)-(1b) given by (4), and $p_{h}$ its finite element approximation given by (5). Let next $\mathbf{t}_{h}$ be given by (6) and let $\mathcal{T}_{h}$ be shape-regular, i.e., $\min _{K \in \mathcal{T}_{h}}|K| / h_{K}^{d} \geq \kappa_{\mathcal{T}}$ for some positive constant $\kappa_{\mathcal{T}}$. Then for the a posteriori error estimators of Theorem 1.2, there holds

$$
\begin{aligned}
\eta_{\mathrm{DF}, D} & \leq C\left\|p-p_{h}\right\|_{D}, \\
\eta_{\mathrm{R}, D} & \leq \tilde{C}\left\|p-p_{h}\right\|_{D},
\end{aligned}
$$

where the constant $C$ depends only on the space dimension $d$ and on the shape regularity parameter $\kappa_{\mathcal{T}}$ and the constant $\tilde{C}$ in addition depends on $C_{\mathrm{P}, D}$ from the Poincaré inequality (8) if $D \in \mathcal{D}_{h}^{\mathrm{int}}$ or on $C_{\mathrm{F}, D, \partial \Omega}$ from the Friedrichs inequality (9) if $D \in \mathcal{D}_{h}^{\text {ext }}$.

\section{Classical Residual estimates with all COnstants evaluated}

In order to compare our results with the residual estimation technique, cf. Verfürth [19], we recall here these standard estimates, with the constants evaluated according to Carstensen and Funken [4, Section 5].

Corollary 2.1 (Classical residual estimates with all constants evaluated). Let $p$ be the weak solution of problem (1a)-(1b) given by (4) and let $p_{h}$ be its finite element approximation given by (5). Then

$$
\left\|p-p_{h}\right\| \leq C_{1}\left\{\sum_{K \in \mathcal{T}_{h}} h_{K}^{2}\left\|f+\triangle p_{h}\right\|_{K}^{2}\right\}^{1 / 2}+C_{2}\left\{\sum_{\sigma \in \mathcal{E}_{h}} h_{\sigma}\left\|\left[\nabla p_{h} \cdot \mathbf{n}\right]\right\|_{\sigma}^{2}\right\}^{1 / 2},
$$

where

$$
\begin{aligned}
& C_{V}:= \begin{cases}C_{\mathrm{P}}^{\frac{1}{2}}, \mathcal{T}_{V} h_{\mathcal{T}_{V}} & V \in \mathcal{V}_{h}^{\text {int }}, \\
C_{\mathrm{F}, \mathcal{T}_{V}, \partial \Omega}^{\frac{1}{2}} h_{\mathcal{T}_{V}} & V \in \mathcal{V}_{h}^{\text {ext }}\end{cases} \\
& C_{1}:=\max _{K \in \mathcal{T}_{h}}\left\{\sum_{V \in \mathcal{V}_{K}} C_{V}^{2} / \min _{K \in \mathcal{T}_{V}} h_{K}^{2}\right\}^{\frac{1}{2}}, \\
& C_{2}^{2}:=3 C_{1} \max _{K \in \mathcal{T}_{h}} \max _{\sigma \in \mathcal{E}_{K}}\left\{h_{K} / h_{\sigma} h_{K}^{2} /|K|\right\}+\frac{1}{2} 3^{\frac{3}{2}} C_{1}^{2} \max _{K \in \mathcal{T}_{h}} \max _{\sigma \in \mathcal{E}_{K}}\left\{h_{K} / h_{\sigma} h_{K}^{2} /|K|\left(3+h_{K}^{2} /|K|\right)\right\} .
\end{aligned}
$$


Let us here compare briefly these and our estimates from the theoretical viewpoint (a numerical comparison is done in Section 4 below):

Remark 2.2 (Comparison of our and classical residual estimates). We point out the following differences between our and the above residual estimates:

- In our approach, the strong form (1a) of the equation is applied to the vector field $\mathbf{t}_{h}$ which has been "made for" ( $\mathbf{t}_{h}$ has the appropriate regularity, i.e., $\mathbf{t}_{h} \in \mathbf{H}(\operatorname{div}, \Omega)$ and is locally conservative by $\left.(10)\right)$ whereas in the classical approach, it is applied directly to $p_{h}$, which has only been designed for the weak form of (1a) given by (4) and which in particular is such that $\Delta p_{h}=0$.

- The constants have the same nature in both estimates: it is basically the Poincaré constant $C_{\mathrm{P}}$ for interior vertices and the Friedrichs constant $C_{\mathrm{F}}$ for exterior vertices.

- There is no "overlapping" in the computation of our constants (as $D \in \mathcal{D}_{h}$ are disjoint), whereas there is an overlapping in the computation of the classical constants (as $\mathcal{T}_{V}, V \in \mathcal{V}_{h}$, are not disjoint).

- There is no maximization over the sides of an element / the whole mesh of the constants and different geometrical quantities in our estimates.

- The constants are very simple in our estimates, whereas the computer evaluation of the constants in the classical estimates is rather complicated.

- One is led to construct the meshes $\mathcal{D}_{h}$ and $\mathcal{S}_{h}$ in our estimates, which is not the case in the classical estimates.

- Our approach extends readily to three ( $d$ in general) space dimensions.

\section{IMPROVEMENTS USING LOCAL MINIMIZATION}

In Section 1, we have shown that a choice of $\mathbf{t}_{h} \in \mathbf{H}(\operatorname{div}, \Omega)$ in Theorem 1.2 leading to local efficiency and robustness with respect to the domain and the weak solution for the finite element method is given by (6). However, it is not apparent at all whether this choice leads to the best upper bound. In particular, by closer investigation, it turns out that whereas in mixed finite element or finite volume (discontinuous Galerkin) methods, the residual estimator represents a higher-order term, as in these methods one has (with an appropriate $\left.\mathbf{t}_{h}\right)\left(\nabla \cdot \mathbf{t}_{h}, 1\right)_{K}=(f, 1)_{K}$ for all $K \in \mathcal{T}_{h}$, which implies $\nabla \cdot \mathbf{t}_{h}=(f, 1)_{K} /|K|$ by the properties of the RaviartThomas-Nédélec elements, it is not the case here, as (7) is only true on a set of elements $\mathcal{S}_{D}(D)$ and not on each element $K \in \mathcal{S}_{D}$. The numerical experiments for $\mathbf{t}_{h}$ given by (6) presented in Section 4 below indeed show that the residual estimators $\eta_{\mathrm{R}, D}$ represent a major contribution to the estimate in the present case.

A natural idea in order to decrease the estimate is to try to choose another $\mathbf{t}_{h} \in \mathbf{H}(\operatorname{div}, \Omega)$ satisfying (10). Notice now that $\mathbf{t}_{h} \in \mathbf{R T N}\left(\mathcal{S}_{h}\right)$ given by (6) only for such $\sigma \in \mathcal{G}_{h}$ which are at the boundary of some $D \in \mathcal{D}_{h}^{\text {int }}$ satisfies $\mathbf{t}_{h} \in \mathbf{H}(\operatorname{div}, \Omega)$ and (10) and we can choose any value of $\mathbf{t}_{h} \cdot \mathbf{n}_{\sigma}$ for the other edges. In particular, we can choose values that minimize the estimate. Moreover, as the estimator is build locally on each dual volume, we can perform this optimization process locally on each dual volume. We describe here how we perform the minimization in two space dimensions; a similar development can be done in three space dimensions. For the sake of simplicity, we assume henceforth that $f$ is piecewise constant on $\mathcal{T}_{h}$.

\subsection{Local minimization strategy}

Let $D \in \mathcal{D}_{h}$ be the dual volume corresponding to a vertex $V_{D}$ as in Figure $2 ; D$ is decomposed into a subdivision $\mathcal{S}_{D}$ of $n$ subtriangles $K_{0}, \ldots, K_{n-1}$, numbered in the counter-clockwise direction. If $D$ belongs to $\mathcal{D}_{h}^{\text {ext }}$, the triangles $K_{0}$ and $K_{n-1}$ are on the boundary, unambiguously defined by the counter-clockwise order. By construction of the dual volumes, the number $n$ of subtriangles is an even number and $n \geq 6$ for an interior dual volume, $n \geq 2$ for a boundary dual volume. On each subtriangle $K_{i}$, the vertex 0 is the center of the volume $D$, the other vertices are numbered in the counter-clockwise direction, and we call $\sigma_{j}^{i}$ the edge opposite to the vertex $j$. We call $\mathbf{n}_{\sigma_{j}^{i}}$ the exterior normal vector of the edge $\sigma_{j}^{i}$. Let next $\boldsymbol{\psi}_{j}^{i}, j=0,1,2$, be the basis 

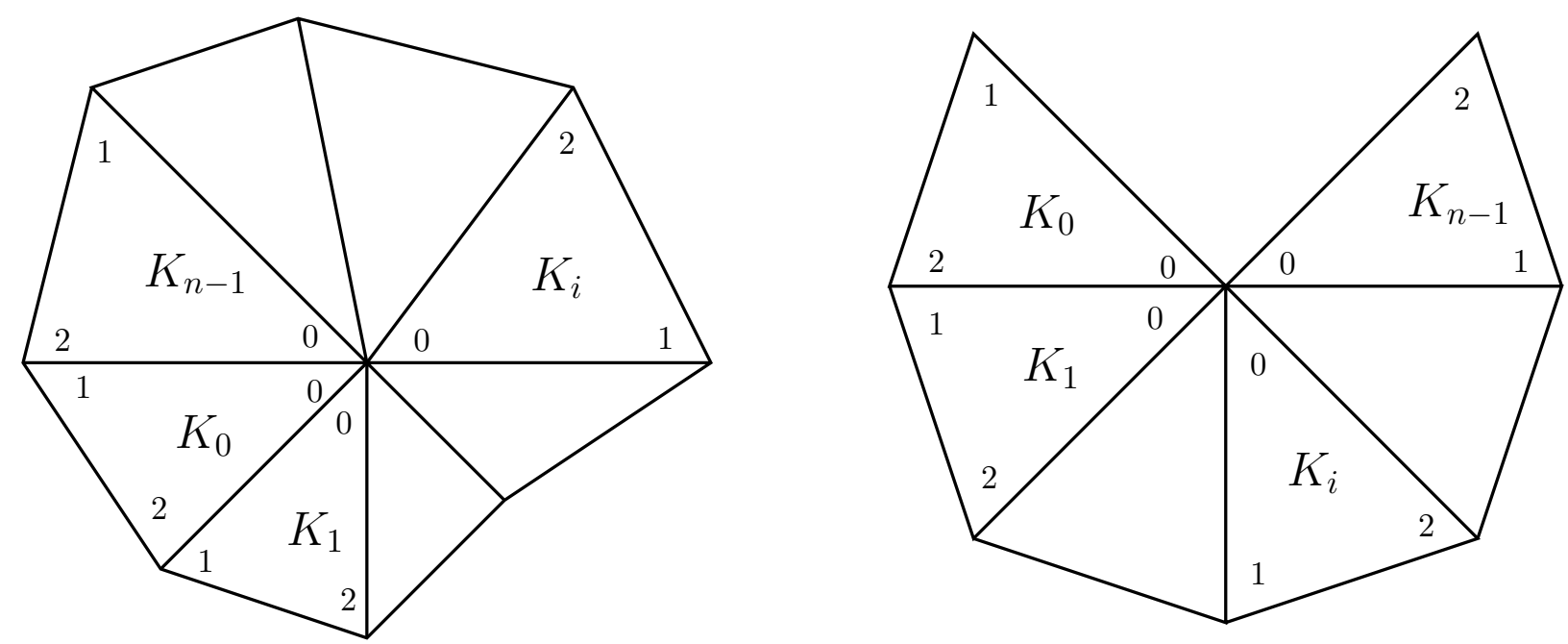

Figure 2. Dual volumes and their subdivisions $\mathcal{S}_{D}$. Left: interior dual volume; right: boundary dual volume

function of $\mathbf{R T N}\left(K_{i}\right)$ corresponding to the vertex $j$, i.e.,

$$
\psi_{j}^{i}=\frac{1}{d\left|K_{i}\right|}\left(\mathbf{x}-V_{j}^{i}\right)
$$

where $V_{j}^{i}$ is vertex $j$ of the triangle $K_{i}$. On $K_{i}, \mathbf{t}_{h}$ can consequently be written as

$$
\left.\mathbf{t}_{h}\right|_{K_{i}}=\alpha_{0}^{i} \psi_{0}^{i}+\alpha_{1}^{i} \psi_{1}^{i}+\alpha_{2}^{i} \psi_{2}^{i}
$$

The values of the external fluxes over $\partial D$ are prescribed by (6) in the same way as before:

- for any dual volume $D \in \mathcal{D}_{h}$,

$$
\alpha_{0}^{i}=-\left|\sigma_{0}^{i}\right| \nabla p_{h} \cdot \mathbf{n}_{\sigma_{0}^{i}}, i=0, \ldots, n-1
$$

- if $D \in \mathcal{D}_{h}^{\text {ext }}$, then in addition

$$
\alpha_{2}^{0}=-\left|\sigma_{2}^{0}\right| \nabla p_{h} \cdot \mathbf{n}_{\sigma_{2}^{0}} \text { and } \alpha_{1}^{n-1}=-\left|\sigma_{1}^{n-1}\right| \nabla p_{h} \cdot \mathbf{n}_{\sigma_{1}^{n-1}}
$$

The internal fluxes, given by the coefficients $\alpha_{1}^{i}$ and $\alpha_{2}^{i}$, have to first fulfill the continuity of the normal trace across the edges, which imposes

- if $D \in \mathcal{D}_{h}^{\text {int }}$,

$$
\alpha_{1}^{i}+\alpha_{2}^{i+1}=0, i=0, \ldots, n-1 \text { with } \alpha_{2}^{n}=\alpha_{2}^{0}
$$

- if $D \in \mathcal{D}_{h}^{\text {ext }}$,

$$
\alpha_{1}^{i}+\alpha_{2}^{i+1}=0, i=0, \ldots, n-2 .
$$

Therefore, there are $n$ degrees of freedom $\alpha^{i}=\alpha_{1}^{i}, i=0, \ldots, n-1$, if $D \in \mathcal{D}_{h}^{\text {int }}$, and $n-1$ degrees of freedom $\alpha^{i}=\alpha_{1}^{i}, i=0, \ldots, n-2$, if $D \in \mathcal{D}_{h}^{\text {ext }}$ left and these can be chosen in order to minimize the estimator; from now on, the local estimator on a given $D$ will be considered as a function of them. 
We denote $\mathbf{X}=\left(\alpha^{0}, \ldots, \alpha^{n-1}\right)^{t}$ if $D \in \mathcal{D}_{h}^{\text {int }}$ and $\mathbf{X}=\left(\alpha^{0}, \ldots, \alpha^{n-2}\right)^{t}$ if $D \in \mathcal{D}_{h}^{\text {ext }}$ and write

$$
\eta_{D}^{2}(\mathbf{X})=\left(\eta_{\mathrm{DF}, D}(\mathbf{X})+\eta_{\mathrm{R}, D}(\mathbf{X})\right)^{2}
$$

We seek the minimized estimator

$$
\eta_{D}^{\min }=\min _{\mathbf{X}} \eta_{D}(\mathbf{X})
$$

and redefine consequently our estimate as

$$
\eta:=\left\{\sum_{D \in \mathcal{D}_{h}}\left(\eta_{D}^{\min }\right)^{2}\right\}^{1 / 2}
$$

In fact, as we are looking for a minimization strategy that would not increase too much the computational cost of the estimator, we rather choose to minimize

$$
\eta_{\mathrm{DF}, D}^{2}(\mathbf{X})+\eta_{\mathrm{R}, D}^{2}(\mathbf{X})
$$

instead of $\eta_{D}^{2}(\mathbf{X})$. In contrast to $\eta_{D}^{2}(\mathbf{X})$, we will see that this expression is a quadratic form in terms of $\mathbf{X}$.

Finally, note once again that on a given dual volume $D \in \mathcal{D}_{h}$, the coefficients $\alpha_{1}^{i}$ and $\alpha_{2}^{i}$ correspond to internal edges (except $\alpha_{2}^{0}$ and $\alpha_{1}^{n-1}$ if $D \in \mathcal{D}_{h}^{\text {ext }}$ ); consequently, if we modify them, we modify the local estimator $\eta_{D}$ but not the local estimator in the other dual volumes. As the minimization process will be done with respect to these coefficients, this means that we can perform it locally, independently on each dual volume.

We now express the local estimators with respect to the degrees of freedom, first for interior and then for boundary dual volumes.

\subsection{Estimators for interior dual volumes}

Let $D \in \mathcal{D}_{h}^{\text {int }}$ be an interior dual volume and $\mathcal{S}_{D}=\left\{K_{0}, \ldots, K_{n-1}\right\}$ its subtriangulation. Using condition (14), we obtain

$$
\begin{aligned}
\left.\mathbf{t}_{h}\right|_{K_{0}} & =\alpha_{0}^{0} \boldsymbol{\psi}_{0}^{0}+\alpha^{0} \boldsymbol{\psi}_{1}^{0}-\alpha^{n-1} \boldsymbol{\psi}_{2}^{0}, \\
\left.\mathbf{t}_{h}\right|_{K_{i}} & =\alpha_{0}^{i} \boldsymbol{\psi}_{0}^{i}+\alpha^{i} \boldsymbol{\psi}_{1}^{i}-\alpha^{i-1} \boldsymbol{\psi}_{2}^{i}, \quad i=1, \ldots, n-1 .
\end{aligned}
$$

We now calculate separately the residual and the diffusive flux estimators.

\subsubsection{Residual estimator}

For each dual volume $D \in \mathcal{D}_{h}^{\text {int }}$, the residual estimator is defined as

$$
\begin{aligned}
\eta_{\mathrm{R}, D}^{2} & =m_{D}^{2}\left\|f-\nabla \cdot \mathbf{t}_{h}\right\|_{D}^{2}=m_{D}^{2} \sum_{K \in \mathcal{S}_{D}}\left\|f-\nabla \cdot \mathbf{t}_{h}\right\|_{K}^{2} \\
& =m_{D}^{2} \sum_{K \in \mathcal{S}_{D}} \int_{K}\left(f-\nabla \cdot \mathbf{t}_{h}\right)^{2} \mathrm{~d} \mathbf{x} .
\end{aligned}
$$

To evaluate the above integrals over triangles, as well as similarly below, we will use the quadrature formula

$$
\int_{K} g \mathrm{~d} \mathbf{x} \approx \frac{|K|}{3} \sum_{\sigma \in \mathcal{E}_{K}} g\left(M_{\sigma}\right),
$$

where $M_{\sigma}$ is the midpoint of the edge $\sigma$, which is exact for polynomials of second order.

Using (16) and the definition of $\boldsymbol{\psi}_{j}^{i}$, we have

$$
\begin{aligned}
& \left.\mathbf{t}_{h}\right|_{K_{0}}=\frac{1}{d\left|K_{0}\right|}\left(\alpha_{0}^{0}\left(\mathbf{x}-V_{0}^{0}\right)+\alpha^{0}\left(\mathbf{x}-V_{1}^{0}\right)-\alpha^{n-1}\left(\mathbf{x}-V_{2}^{0}\right)\right) \\
& \left.\mathbf{t}_{h}\right|_{K_{i}}=\frac{1}{d\left|K_{i}\right|}\left(\alpha_{0}^{i}\left(\mathbf{x}-V_{0}^{i}\right)+\alpha^{i}\left(\mathbf{x}-V_{1}^{i}\right)-\alpha^{i-1}\left(\mathbf{x}-V_{2}^{i}\right)\right), \quad i=1, \ldots, n-1
\end{aligned}
$$


and

$$
\begin{aligned}
\left.\nabla \cdot \mathbf{t}_{h}\right|_{K_{0}} & =\frac{1}{\left|K_{0}\right|}\left(\alpha_{0}^{0}+\alpha^{0}-\alpha^{n-1}\right), \\
\left.\nabla \cdot \mathbf{t}_{h}\right|_{K_{i}} & =\frac{1}{\left|K_{i}\right|}\left(\alpha_{0}^{i}+\alpha^{i}-\alpha^{i-1}\right), \quad i=1, \ldots, n-1 .
\end{aligned}
$$

Using (19) and denoting $f_{j}^{i}:=\left|K_{i}\right| f\left(M_{\sigma_{j}^{i}}\right)$, we have

$$
\begin{aligned}
& m_{D}^{2} \int_{K_{0}}\left(f-\nabla \cdot \mathbf{t}_{h}\right)^{2} \mathrm{~d} \mathbf{x}=C_{0}^{0}+C_{1}^{0} \alpha^{0}+C_{2}^{0} \alpha^{n-1}+C_{3}^{0} \alpha^{n-1} \alpha^{0}+C_{4}^{0}\left(\alpha^{0}\right)^{2}+C_{5}^{0}\left(\alpha^{n-1}\right)^{2}, \\
& m_{D}^{2} \int_{K_{i}}\left(f-\nabla \cdot \mathbf{t}_{h}\right)^{2} \mathrm{~d} \mathbf{x}=C_{0}^{i}+C_{1}^{i} \alpha^{i}+C_{2}^{i} \alpha^{i-1}+C_{3}^{i} \alpha^{i-1} \alpha^{i}+C_{4}^{i}\left(\alpha^{i}\right)^{2}+C_{5}^{i}\left(\alpha^{i-1}\right)^{2}, \quad i=1, \ldots, n-1,
\end{aligned}
$$

where, for $i=0, \ldots, n$ :

$$
\begin{aligned}
C_{0}^{i} & =\frac{m_{D}^{2}}{3\left|K_{i}\right|}\left[\left(f_{0}^{i}\right)^{2}+\left(f_{1}^{i}\right)^{2}+\left(f_{2}^{i}\right)^{2}-2\left(f_{0}^{i}+f_{1}^{i}+f_{2}^{i}\right) \alpha_{0}^{i}+3\left(\alpha_{0}^{i}\right)^{2}\right] \\
C_{1}^{i} & =\frac{m_{D}^{2}}{3\left|K_{i}\right|}\left[-2\left(f_{0}^{i}+f_{1}^{i}+f_{2}^{i}\right)+6 \alpha_{0}^{i}\right] \\
C_{2}^{i} & =-C_{1}^{i}, \\
C_{3}^{i} & =\frac{-2 m_{D}^{2}}{\left|K_{i}\right|} \\
C_{4}^{i} & =\frac{m_{D}^{2}}{\left|K_{i}\right|} \\
C_{5}^{i} & =\frac{m_{D}^{2}}{\left|K_{i}\right|}
\end{aligned}
$$

Thus the residual estimator writes

$$
\begin{aligned}
\eta_{\mathrm{R}, D}^{2}= & \sum_{i=0}^{n-1} \int_{K_{i}}\left(f-\nabla \cdot \mathbf{t}_{h}\right)^{2} \mathrm{~d} \mathbf{x} \\
= & \sum_{i=0}^{n-1} C_{0}^{i}+\sum_{i=0}^{n-2}\left(C_{1}^{i}+C_{2}^{i+1}\right) \alpha^{i}+\left(C_{1}^{n-1}+C_{2}^{0}\right) \alpha^{n-1} \\
& +C_{3}^{0} \alpha^{0} \alpha^{n-1}+C_{4}^{0}\left(\alpha^{0}\right)^{2}+C_{5}^{0}\left(\alpha^{n-1}\right)^{2}+\sum_{i=1}^{n-1}\left(C_{3}^{i} \alpha^{i} \alpha^{i-1}+C_{4}^{i}\left(\alpha^{i}\right)^{2}+C_{5}^{i}\left(\alpha^{i-1}\right)^{2}\right)
\end{aligned}
$$

and we notice that $\eta_{\mathrm{R}, D}^{2}$ is a quadratic form with respect to $\mathbf{X}=\left(\alpha^{0}, \ldots, \alpha^{n-1}\right)^{t}$ :

$$
\eta_{\mathrm{R}, D}^{2}(\mathbf{X})=a_{\mathrm{R}}-\mathbf{B}_{\mathrm{R}}^{t} \mathbf{X}+\frac{1}{2} \mathbf{X}^{t} \mathbb{A}_{\mathrm{R}} \mathbf{X},
$$

where

$$
a_{\mathrm{R}}=\sum_{i=0}^{n-1} C_{0}^{i}, \quad \mathbf{B}_{\mathrm{R}}=-\left(\begin{array}{c}
C_{1}^{0}+C_{2}^{1} \\
\vdots \\
C_{1}^{n-1}+C_{2}^{0}
\end{array}\right), \quad \mathbb{A}_{\mathrm{R}}=\left(\begin{array}{ccccc}
2\left(C_{4}^{0}+C_{5}^{1}\right) & C_{3}^{1} & & & C_{3}^{0} \\
C_{3}^{1} & \ddots & \ddots & & \\
& \ddots & \ddots & \ddots & \\
& & \ddots & \ddots & C_{3}^{n-1} \\
& & & C_{3}^{n-1} & 2\left(C_{4}^{n-1}+C_{5}^{0}\right)
\end{array}\right) .
$$

\subsubsection{Diffusive flux estimator}

Writting $\mathbf{t}_{h}=\left(\mathbf{t}_{h}^{x}, \mathbf{t}_{h}^{y}\right)^{t}$, the diffusive flux estimator on an interior dual volume $D \in \mathcal{D}_{h}^{\text {int }}$ is given by

$$
\begin{aligned}
\eta_{\mathrm{DF}, D}^{2} & =\left\|\nabla p_{h}+\mathbf{t}_{h}\right\|_{D}^{2} \\
& =\sum_{K \in \mathcal{S}_{D}} \int_{K}\left(\left(\partial_{x} p_{h}+\mathbf{t}_{h}^{x}\right)^{2}+\left(\partial_{y} p_{h}+\mathbf{t}_{h}^{y}\right)^{2}\right) \mathrm{d} \mathbf{x} .
\end{aligned}
$$

We evaluate the integrals over triangles with the quadrature formula (17); using (18) and the fact that $\nabla p_{h}$ is constant over each triangle, we obtain in the same way as for the residual estimator,

$$
\begin{aligned}
\int_{K_{0}}\left(\left(\partial_{x} p_{h}+\mathbf{t}_{h}^{x}\right)^{2}+\left(\partial_{y} p_{h}+\mathbf{t}_{h}^{y}\right)^{2}\right) \mathrm{d} \mathbf{x}= & D_{0}^{0}+D_{1}^{0} \alpha^{0}+D_{2}^{0} \alpha^{n-1}+D_{3}^{0} \alpha^{0} \alpha^{n-1}+D_{4}^{0}\left(\alpha^{0}\right)^{2}+D_{5}^{0}\left(\alpha^{n-1}\right)^{2}, \\
\int_{K_{i}}\left(\left(\partial_{x} p_{h}+\mathbf{t}_{h}^{x}\right)^{2}+\left(\partial_{y} p_{h}+\mathbf{t}_{h}^{y}\right)^{2}\right) \mathrm{d} \mathbf{x}= & D_{0}^{i}+D_{1}^{i} \alpha^{i}+D_{2}^{i} \alpha^{i-1}+D_{3}^{i} \alpha^{i} \alpha^{i-1} \\
& +D_{4}^{i}\left(\alpha^{i}\right)^{2}+D_{5}^{i}\left(\alpha^{i-1}\right)^{2}, \quad i=1, \ldots, n-1,
\end{aligned}
$$


where

$$
\begin{aligned}
D_{0}^{i} & =\frac{1}{3 d^{2}\left|K_{i}\right|} \sum_{j=0}^{2}\left(d\left|K_{i}\right| \partial_{x} p_{h}+\alpha_{0}^{i}\left(m_{j}^{i}-x_{0}^{i}\right)\right)^{2}+\left(d\left|K_{i}\right| \partial_{y} p_{h}+\alpha_{0}^{i}\left(n_{j}^{i}-y_{0}^{i}\right)\right)^{2} \\
D_{1}^{i} & =\frac{2}{3 d^{2}\left|K_{i}\right|} \sum_{j=0}^{2}\left(d\left|K_{i}\right| \partial_{x} p_{h}+\alpha_{0}^{i}\left(m_{j}^{i}-x_{0}^{i}\right)\right)\left(m_{j}^{i}-x_{1}^{i}\right)+\left(d\left|K_{i}\right| \partial_{y} p_{h}+\alpha_{0}^{i}\left(n_{j}^{i}-y_{0}^{i}\right)\right)\left(n_{j}^{i}-y_{1}^{i}\right), \\
D_{2}^{i} & =\frac{2}{3 d^{2}\left|K_{i}\right|} \sum_{j=0}^{2}-\left(d\left|K_{i}\right| \partial_{x} p_{h}+\alpha_{0}^{i}\left(m_{j}^{i}-x_{0}^{i}\right)\right)\left(m_{j}^{i}-x_{2}^{i}\right)-\left(d\left|K_{i}\right| \partial_{y} p_{h}+\alpha_{0}^{i}\left(n_{j}^{i}-y_{0}^{i}\right)\right)\left(n_{j}^{i}-y_{2}^{i}\right), \\
D_{3}^{i} & =\frac{2}{3 d^{2}\left|K_{i}\right|} \sum_{j=0}^{2}-\left(m_{j}^{i}-x_{1}^{i}\right)\left(m_{j}^{i}-x_{2}^{i}\right)-\left(n_{j}^{i}-y_{1}^{i}\right)\left(n_{j}^{i}-y_{2}^{i}\right), \\
D_{4}^{i} & =\frac{1}{3 d^{2}\left|K_{i}\right|} \sum_{j=0}^{2}\left(m_{j}^{i}-x_{1}^{i}\right)^{2}+\left(n_{j}^{i}-y_{1}^{i}\right)^{2} \\
D_{5}^{i} & =\frac{1}{3 d^{2}\left|K_{i}\right|} \sum_{j=0}^{2}\left(m_{j}^{i}-x_{2}^{i}\right)^{2}+\left(n_{j}^{i}-y_{2}^{i}\right)^{2}
\end{aligned}
$$

and $\left(m_{j}^{i}, n_{j}^{i}\right)^{t}=M_{\sigma_{j}^{i}}$ and $\left(x_{j}^{i}, y_{j}^{i}\right)^{t}=V_{j}^{i}$ for $i=0, \ldots, n-1$ and $j=0,1,2$, respectively.

Thus the diffusive flux estimator writes

$$
\begin{aligned}
\eta_{\mathrm{DF}, D}^{2}= & \sum_{i=0}^{n-1} \int_{K_{i}}\left(\left(\partial_{x} p_{h}+\mathbf{t}_{h}^{x}\right)^{2}+\left(\partial_{y} p_{h}+\mathbf{t}_{h}^{y}\right)^{2}\right) \mathrm{d} \mathbf{x} \\
= & \sum_{i=0}^{n-1} D_{0}^{i}+\sum_{i=0}^{n-2}\left(D_{1}^{i}+D_{2}^{i+1}\right) \alpha^{i}+\left(D_{1}^{n-1}+D_{2}^{0}\right) \alpha^{n-1} \\
& +D_{3}^{0} \alpha^{0} \alpha^{n-1}+D_{4}^{0}\left(\alpha^{0}\right)^{2}+D_{5}^{0}\left(\alpha^{n-1}\right)^{2}+\sum_{i=1}^{n-1}\left(D_{3}^{i} \alpha^{i} \alpha^{i-1}+D_{4}^{i}\left(\alpha^{i}\right)^{2}+D_{5}^{i}\left(\alpha^{i-1}\right)^{2}\right)
\end{aligned}
$$

and $\eta_{\mathrm{DF}, D}^{2}$ is a quadratic form with respect to $\mathbf{X}=\left(\alpha^{0}, \ldots, \alpha^{n-1}\right)^{t}$ :

$$
\eta_{\mathrm{DF}, D}^{2}(\mathbf{X})=a_{\mathrm{DF}}-\mathbf{B}_{\mathrm{DF}}^{t} \mathbf{X}+\frac{1}{2} \mathbf{X}^{t} \mathbb{A}_{\mathrm{DF}} \mathbf{X}
$$

where

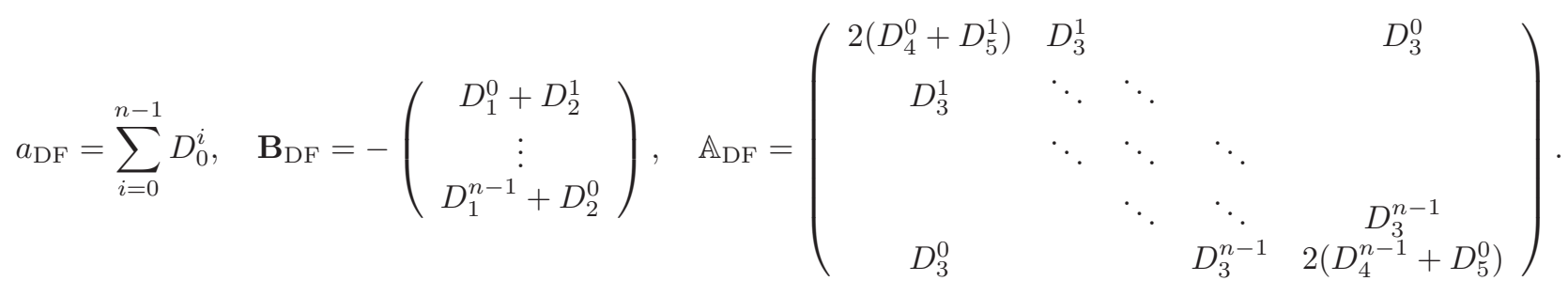

\subsection{Estimators for boundary dual volumes}

Let $D \in \mathcal{D}_{h}^{\text {ext }}$ be a boundary dual volume, and $\mathcal{S}_{D}=\left\{K_{0}, \ldots, K_{n-1}\right\}$ its subtriangulation. We recall that now, $\alpha_{2}^{0}$ and $\alpha_{1}^{n-1}$ are already prescribed and considered as constants, and that the degrees of freedom are $\mathbf{X}=\left(\alpha^{0}, \ldots, \alpha^{n-2}\right)^{t}$. Using condition (15), we obtain

$$
\begin{aligned}
\left.\mathbf{t}_{h}\right|_{K_{0}} & =\alpha_{0}^{0} \boldsymbol{\psi}_{0}^{0}+\alpha^{0} \boldsymbol{\psi}_{1}^{0}+\alpha_{2}^{0} \boldsymbol{\psi}_{2}^{0}, \\
\left.\mathbf{t}_{h}\right|_{K_{i}} & =\alpha_{0}^{i} \boldsymbol{\psi}_{0}^{i}+\alpha^{i} \boldsymbol{\psi}_{1}^{i}-\alpha^{i-1} \boldsymbol{\psi}_{2}^{i}, \quad i=1, \ldots, n-2 .
\end{aligned}
$$

Unlike interior dual volumes, boundary dual volumes can have only two subtriangles; this limit case has to be taken care of separately.

\subsubsection{Residual estimator}

We distinguish two cases.

- General case $n>2$ :

We find

$$
\begin{aligned}
\eta_{\mathrm{R}, D}^{2}= & \tilde{C}_{0}^{0}+\sum_{i=1}^{n-3} C_{0}^{i}+\tilde{C}_{0}^{n-2}+\left(\tilde{C}_{1}^{0}+C_{2}^{1}\right) \alpha^{0}+\sum_{i=1}^{n-3}\left(C_{1}^{i}+C_{2}^{i+1}\right) \alpha^{i}+\tilde{C}_{1}^{n-2} \alpha^{n-2} \\
& +C_{4}^{0}\left(\alpha^{0}\right)^{2}+\sum_{i=1}^{n-3}\left(C_{3}^{i} \alpha^{i-1} \alpha^{i}+C_{4}^{i}\left(\alpha^{i}\right)^{2}+C_{5}^{i}\left(\alpha^{i-1}\right)^{2}\right) \\
& +C_{3}^{n-2} \alpha^{n-3} \alpha^{n-2}+\tilde{C}_{4}^{n-2}\left(\alpha^{n-2}\right)^{2}+C_{5}^{n-2}\left(\alpha^{n-3}\right)^{2}
\end{aligned}
$$


where

$$
\begin{aligned}
\tilde{C}_{0}^{0} & =C_{0}^{0}-C_{2}^{0} \alpha_{2}^{0}+C_{5}^{0}\left(\alpha_{2}^{0}\right)^{2}, \\
\tilde{C}_{1}^{0} & =C_{1}^{0}-C_{3}^{0} \alpha_{2}^{0}, \\
\tilde{C}_{0}^{n-2} & =C_{0}^{n-2}+C_{0}^{n-1}+C_{1}^{n-1} \alpha_{1}^{n-1}+C_{4}^{n-1}\left(\alpha_{1}^{n-1}\right)^{2}, \\
\tilde{C}_{1}^{n-2} & =C_{1}^{n-2}+C_{2}^{n-1}+C_{3}^{n-1} \alpha_{1}^{n-1}, \\
\tilde{C}_{4}^{n-2} & =C_{4}^{n-2}+C_{5}^{n-1} .
\end{aligned}
$$

Once again, $\eta_{\mathrm{R}, D}^{2}$ is a quadratic form with respect to $\mathbf{X}=\left(\alpha^{0}, \ldots, \alpha^{n-2}\right)^{t}$ :

$$
\eta_{\mathrm{R}, D}^{2}(\mathbf{X})=a_{\mathrm{R}}-\mathbf{B}_{\mathrm{R}}^{t} \mathbf{X}+\frac{1}{2} \mathbf{X}^{t} \mathbb{A}_{\mathrm{R}} \mathbf{X}
$$

where

$$
\begin{aligned}
& a_{\mathrm{R}}=\tilde{C}_{0}^{0}+\sum_{i=1}^{n-3} C_{0}^{i}+\tilde{C}_{0}^{n-2}, \quad \mathbf{B}_{\mathrm{R}}=-\left(\begin{array}{c}
\tilde{C}_{1}^{0}+C_{2}^{1} \\
C_{1}^{1}+C_{2}^{2} \\
\vdots \\
C_{1}^{n-3}+C_{2}^{n-2} \\
\tilde{C}_{1}^{n-2}
\end{array}\right), \\
& \mathbb{A}_{\mathrm{R}}=\left(\begin{array}{ccccc}
2\left(C_{4}^{0}+C_{5}^{1}\right) & C_{3}^{1} & & \\
C_{3}^{1} & \ddots & \ddots & & \\
& \ddots & \ddots & \ddots & \\
& & \ddots & 2\left(C_{4}^{n-3}+C_{5}^{n-2}\right) & C_{3}^{n-2} \\
& & & C_{3}^{n-2} & 2 \tilde{C}_{4}^{n-2}
\end{array}\right) .
\end{aligned}
$$

- Limit case $n=2$ :

There is only one degree of freedom $x=\alpha^{0}$ and we find

$$
\eta_{\mathrm{R}, D}^{2}=a_{\mathrm{R}} x^{2}+b_{\mathrm{R}} x+c_{\mathrm{R}}
$$

where

$$
\begin{aligned}
a_{\mathrm{R}} & =C_{4}^{0}+C_{5}^{1}, \\
b_{\mathrm{R}} & =C_{1}^{0}+C_{2}^{1}-C_{3}^{0} \alpha_{2}^{0}+C_{3}^{1} \alpha_{1}^{1}, \\
c_{\mathrm{R}} & =C_{0}^{0}+C_{1}^{1} \alpha_{1}^{0}-C_{2}^{0} \alpha_{2}^{0}+C_{5}^{0}\left(\alpha_{2}^{0}\right)^{2}+C_{4}^{1}\left(\alpha_{1}^{1}\right)^{2} .
\end{aligned}
$$

3.3.2. Diffusive flux estimator

We distinguish two cases.

- General case $n>2$ :

We find

$$
\begin{aligned}
\eta_{\mathrm{DF}, D}^{2}= & \tilde{D}_{0}^{0}+\sum_{i=1}^{n-3} D_{0}^{i}+\tilde{D}_{0}^{n-2}+\left(\tilde{D}_{1}^{0}+D_{2}^{1}\right) \alpha^{0}+\sum_{i=1}^{n-3}\left(D_{1}^{i}+D_{2}^{i+1}\right) \alpha^{i}+\tilde{D}_{1}^{n-2} \alpha^{n-2} \\
& +D_{4}^{0}\left(\alpha^{0}\right)^{2}+\sum_{i=1}^{n-3}\left(D_{3}^{i} \alpha^{i-1} \alpha^{i}+D_{4}^{i}\left(\alpha^{i}\right)^{2}+D_{5}^{i}\left(\alpha^{i-1}\right)^{2}\right) \\
& +D_{3}^{n-2} \alpha^{n-3} \alpha^{n-2}+\tilde{D}_{4}^{n-2}\left(\alpha^{n-2}\right)^{2}+D_{5}^{n-2}\left(\alpha^{n-3}\right)^{2}
\end{aligned}
$$

where

$$
\begin{aligned}
\tilde{D}_{0}^{0} & =D_{0}^{0}-D_{2}^{0} \alpha_{2}^{0}+D_{5}^{0}\left(\alpha_{2}^{0}\right)^{2} \\
\tilde{D}_{1}^{0} & =D_{1}^{0}-D_{3}^{0} \alpha_{2}^{0} \\
\tilde{D}_{0}^{n-2} & =D_{0}^{n-2}+D_{0}^{n-1}+D_{1}^{n-1} \alpha_{1}^{n-1}+D_{4}^{n-1}\left(\alpha_{1}^{n-1}\right)^{2} \\
\tilde{D}_{1}^{n-2} & =D_{1}^{n-2}+D_{2}^{n-1}+D_{3}^{n-1} \alpha_{1}^{n-1} \\
\tilde{D}_{4}^{n-2} & =D_{4}^{n-2}+D_{5}^{n-1}
\end{aligned}
$$


Once again, $\eta_{\mathrm{DF}, D}^{2}$ is a quadratic form with respect to $\mathbf{X}=\left(\alpha^{0}, \ldots, \alpha^{n-2}\right)^{t}$ :

$$
\eta_{\mathrm{DF}, D}^{2}(\mathbf{X})=a_{\mathrm{DF}}-\mathbf{B}_{\mathrm{DF}}^{t} \mathbf{X}+\frac{1}{2} \mathbf{X}^{t} \mathbb{A}_{\mathrm{DF}} \mathbf{X}
$$

where

$$
\begin{aligned}
& a_{\mathrm{DF}}=\tilde{D}_{0}^{0}+\sum_{i=1}^{n-3} D_{0}^{i}+\tilde{D}_{0}^{n-2}, \mathbf{B}_{\mathrm{DF}}=-\left(\begin{array}{c}
\tilde{D}_{1}^{0}+D_{2}^{1} \\
D_{1}^{1}+D_{2}^{2} \\
\vdots \\
D_{1}^{n-3}+D_{2}^{n-2} \\
\tilde{D}_{1}^{n-2}
\end{array}\right), \\
& \mathbb{A}_{\mathrm{DF}}=\left(\begin{array}{ccccc}
2\left(D_{4}^{0}+D_{5}^{1}\right) & D_{3}^{1} & & \\
D_{3}^{1} & \ddots & \ddots & \\
& \ddots & \ddots & \ddots & \\
& & \ddots & 2\left(D_{4}^{n-3}+D_{5}^{n-2}\right) & D_{3}^{n-2} \\
& & & D_{3}^{n-2} & 2 \tilde{D}_{4}^{n-2}
\end{array}\right) .
\end{aligned}
$$

- Limit case $n=2$ :

We find

$$
\eta_{\mathrm{DF}, D}^{2}=a_{\mathrm{DF}} x^{2}+b_{\mathrm{DF}} x+c_{\mathrm{DF}},
$$

where

$$
\begin{aligned}
& a_{\mathrm{DF}}=D_{4}^{0}+D_{5}^{1} \\
& b_{\mathrm{DF}}=D_{1}^{0}+D_{2}^{1}-D_{3}^{0} \alpha_{2}^{0}+D_{3}^{1} \alpha_{1}^{1} \\
& c_{\mathrm{DF}}=D_{0}^{0}+D_{1}^{1} \alpha_{1}^{0}-D_{2}^{0} \alpha_{2}^{0}+D_{5}^{0}\left(\alpha_{2}^{0}\right)^{2}+D_{4}^{1}\left(\alpha_{1}^{1}\right)^{2} .
\end{aligned}
$$

\subsection{Minimization}

Given a dual volume $D \in \mathcal{D}_{h}$, our purpose now is to find the vector of degrees of freedom $\mathbf{X}_{1}$ such that it minimizes $\eta_{\mathrm{DF}, D}^{2}(\mathbf{X})+\eta_{\mathrm{R}, D}^{2}(\mathbf{X})$ and evaluate the quasi-optimal local estimator as

$$
\eta:=\left\{\sum_{D \in \mathcal{D}_{h}}\left(\eta_{D}^{\min }\right)^{2}\right\}^{1 / 2}, \quad \eta_{D}^{\min }=\eta_{D}\left(\mathbf{X}_{1}\right)
$$

We distinguish two cases.

- General case $n>2$ :

Finding $\mathbf{X}_{1}$ is easy: we have

$$
\eta_{\mathrm{R}, D}^{2}(\mathbf{X})+\eta_{\mathrm{DF}, D}^{2}(\mathbf{X})=a-\mathbf{B}^{t} \mathbf{X}+\frac{1}{2} \mathbf{X}^{t} \mathbb{A} \mathbf{X}
$$

where

$$
a=a_{\mathrm{R}}+a_{\mathrm{DF}}, \quad \mathbf{B}=\mathbf{B}_{\mathrm{R}}+\mathbf{B}_{\mathrm{DF}}, \quad \mathbb{A}=\mathbb{A}_{\mathrm{R}}+\mathbb{A}_{\mathrm{DF}} .
$$

The matrices $\mathbb{A}_{R}$ and $\mathbb{A}_{D F}$ are positive, and so is $\mathbb{A} ; \mathbb{A}$ is also definite, as one can easily prove that $\mathbf{X}^{t} \mathbb{A}_{R} \mathbf{X}$ and $\mathbf{X}^{t} \mathbb{A}_{D F} \mathbf{X}$ cannot be zero at the same time except if $\mathbf{X}=0$. Thus, finding $\mathbf{X}_{1}$ is reduced to solving the linear system $\mathbb{A} \mathbf{X}=\mathbf{B}$.

- Limit case $n=2$ :

There is only one degree of freedom $x=\alpha^{0}$ and

$$
\eta_{\mathrm{R}, D}^{2}(x)+\eta_{\mathrm{DF}, D}^{2}(x)=a x^{2}+b x+c,
$$



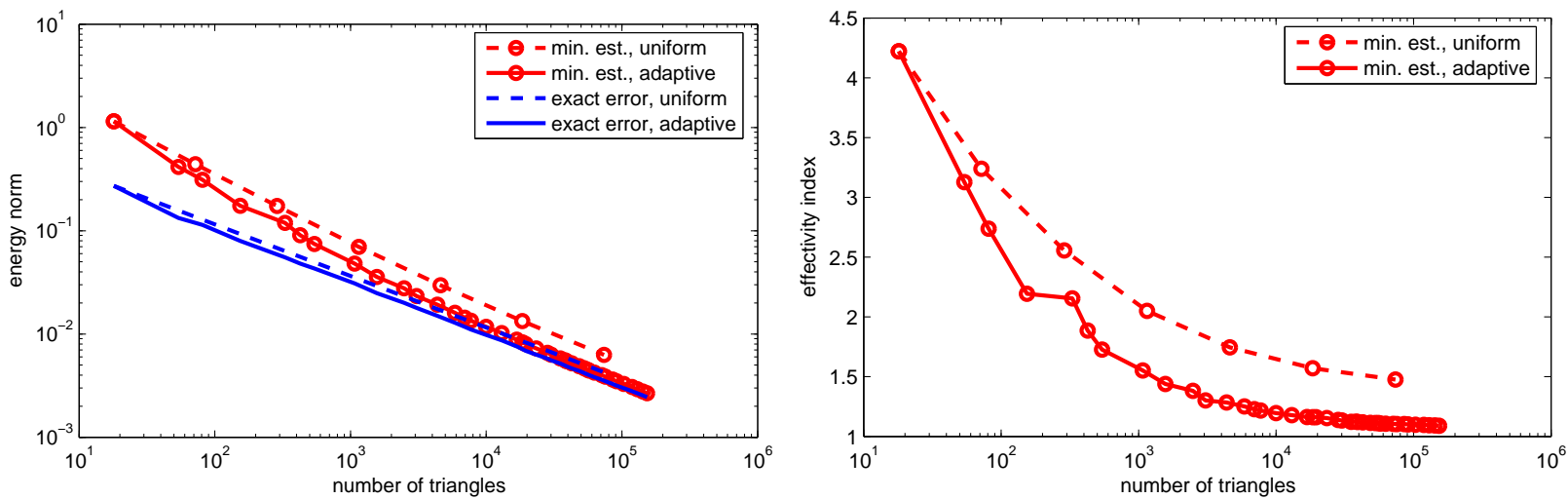

FIGURE 3. Estimated and actual error against the number of elements in uniformly/adaptively refined meshes (left) and corresponding effectivity indices (right) of the minimization estimator $(21)$

where

$$
a=a_{\mathrm{R}}+a_{\mathrm{DF}}, \quad b=b_{\mathrm{R}}+b_{\mathrm{DF}}, \quad c=c_{\mathrm{R}}+c_{\mathrm{DF}} .
$$

The minimum is found for

$$
x=-\frac{b}{2 a} .
$$

Once again, we stress the fact that this minimization process is local and the size of the matrices is small: it corresponds to the number of subtriangles of the dual volume, which is generally of the order of 10 . Thus, the total computational cost of the estimator does not increase excessively and remains linear.

\section{NumericAl RESUlts}

We present here a set of numerical experiments in order to confirm the theoretical results for the estimates of Section 1, demonstrate their improvements using the local minimization of Section 3, and compare these estimates with the residual ones presented in Section 2. We first consider a model problem where the exact solution is smooth, whereas the second problem is posed in a L-shaped domain and has a singular solution. We shall term in this section (12) as the jump estimator, (21) as the minimization estimator, and (13) as the classical residual estimator.

\subsection{Square domain and a regular solution}

We consider here the problem $(1 \mathrm{a})$ on $\Omega=[0,1] \times[0,1]$ with an inhomogeneous Dirichlet boundary condition prescribed instead of $(1 \mathrm{~b})$, given altogether with $f$ by the analytical solution

$$
p=x^{2}+y^{2}+x+y \text {. }
$$

To begin with, we in Figure 3 show the best results we can obtain in this case, using the minimization estimator (21). In particular, in the left part of this figure, the estimated and actual errors are plotted against the number of elements in both uniformly and adaptively refined meshes. As the solution posses no singularity here, the adaptive approach only leads to a slight improvement of the error attained for a given number of unknowns. The effectivity indices are then shown in the right part of Figure 3. We can see that for uniform refinement, we overestimate by a factor of approximately 1.5, whereas for adaptive refinement, this value is very close to the optimal 1 for the finest meshes (1.0376), which suggests the asymptotic exactness of the the minimization estimator in this case. This represents a striking improvement over the classical residual 

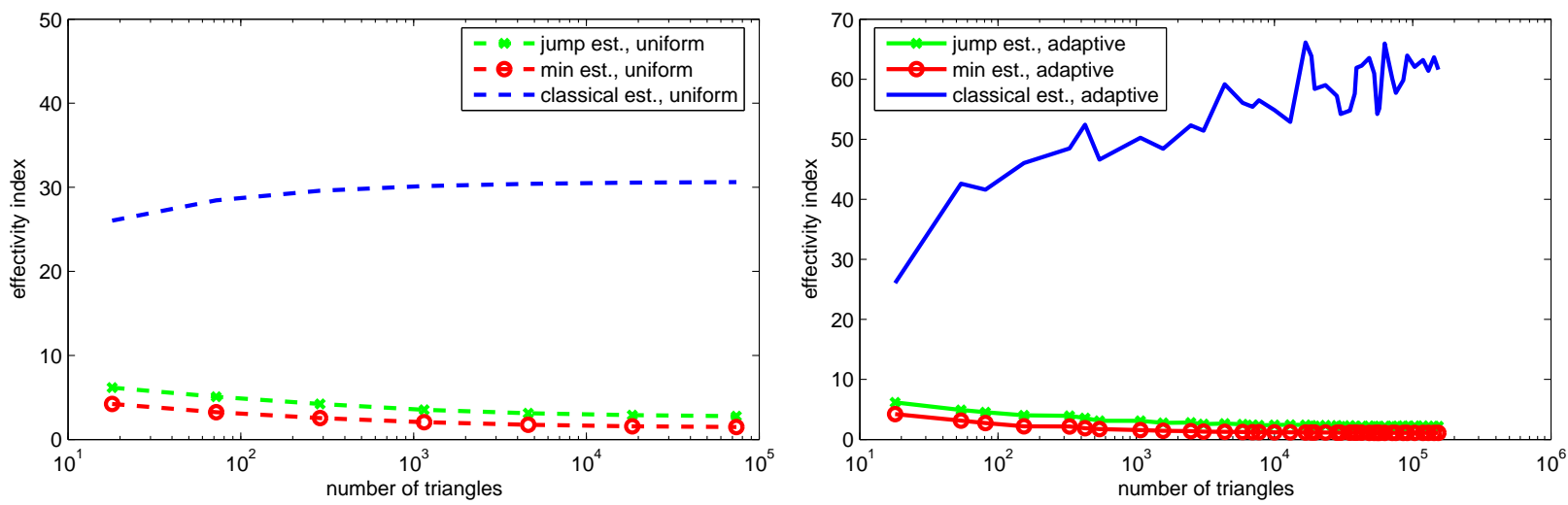

FiguRE 4. Effectivity indices of the jump estimator (12), the minimization estimator (21), and the classical residual estimator (13) against the number of elements in uniformly (left) and adaptively (right) refined meshes
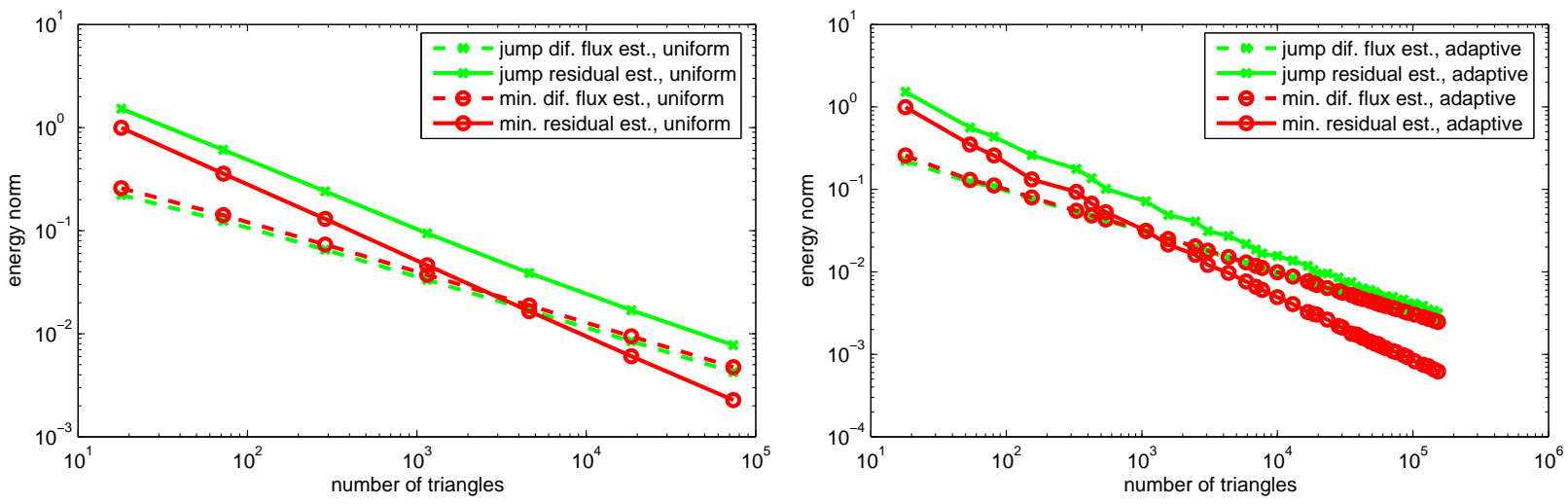

Figure 5. Comparison of the jump estimator (12) and of the minimization estimator (21) components against the number of elements in uniformly (left) and adaptively (right) refined meshes

estimator (13), see Figure 4. The effectivity index of the latter one is around 30 for uniform refinement and around 65 for adaptive refinement. From this figure, we can also see that the original jump estimator (12) performs quite well in this case, with the effectivity indices equal to, respectively, 2.75 and 2 . We finally in Figure 5 compare the diffusive flux and residual parts of the jump and minimization estimators. It turns out that in the jump estimator, the major part of the estimation is represented by the residual estimator and only a minor one by the diffusive flux one. Performing the local minimization of Section 3, the residual estimator gets superconvergent in comparison with the diffusive flux one and consequently insignificant on fine meshes. This is a similar effect as observed before for mixed finite element, finite volume, and discontinuous Galerkin methods in $[7,21,22]$ and we deem it as a prerequisite for asymptotic exactness. 

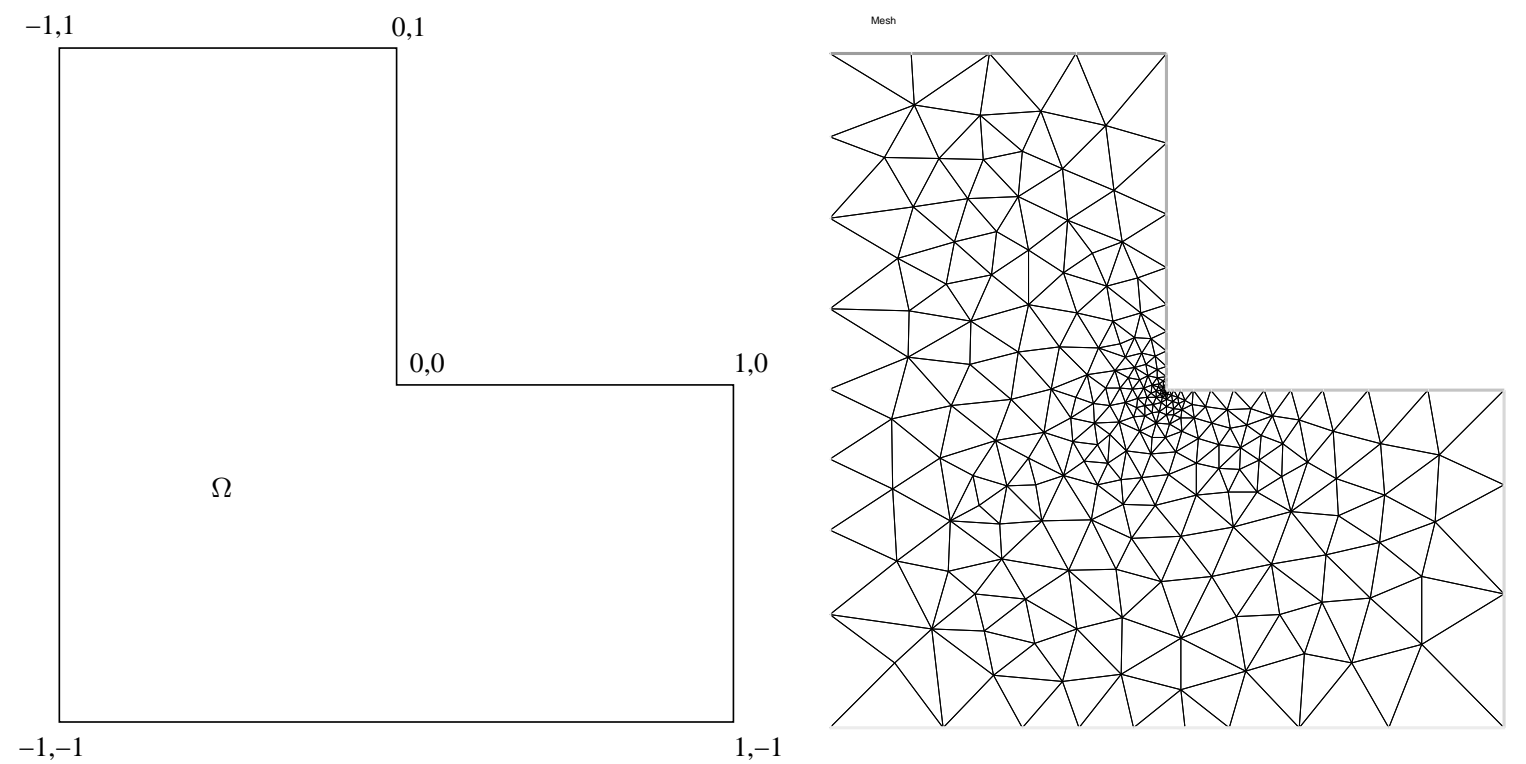

FigURE 6. L-shaped domain geometry (left) and an example of an adaptively refined mesh (right)
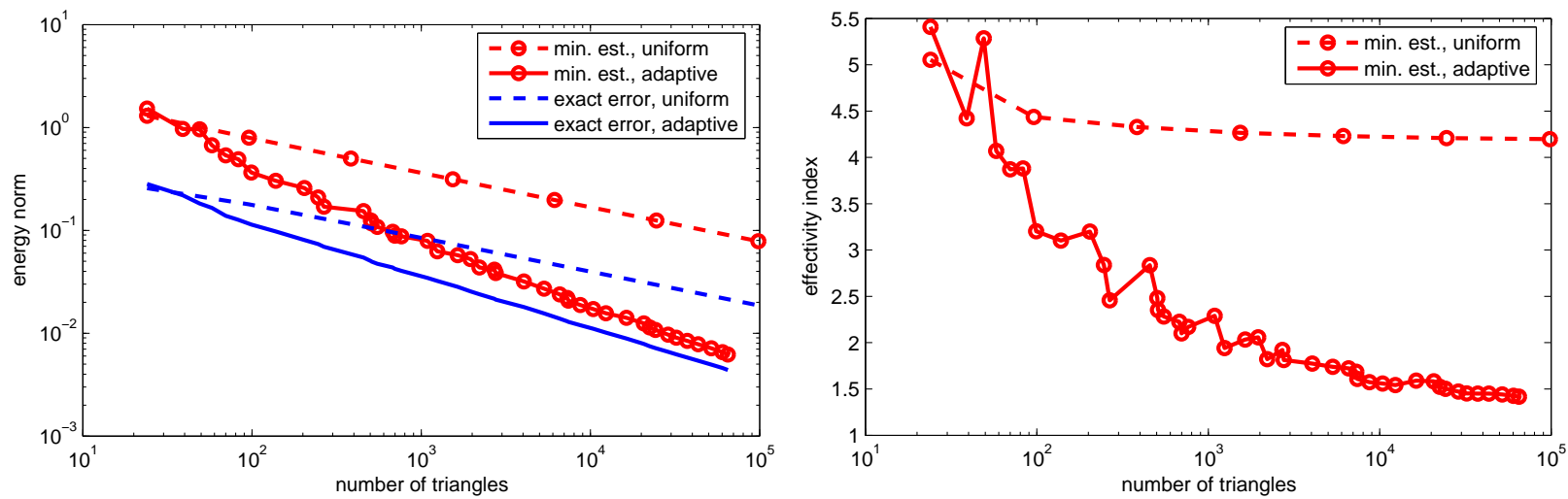

FiguRE 7. Estimated and actual error against the number of elements in uniformly/adaptively refined meshes (left) and corresponding effectivity indices (right) of the minimization estimator $(21)$

\subsection{L-shaped domain and a singular solution}

We consider here the problem (1a) with an inhomogeneous Dirichlet boundary condition prescribed instead of (1b), given altogether with $f$ by the analytical solution

$$
p=r^{\frac{2}{3}} \sin \left(\frac{2}{3} \theta\right) ;
$$

here $(r, \varphi)$ are the polar coordinates. The L-shaped domain $\Omega$ geometry is shown in the left part of Figure 6 .

We again first in Figure 7 show the best results we can obtain in this second case, using the minimization estimator (21). In particular, in the left part of this figure, the estimated and actual errors are plotted against 

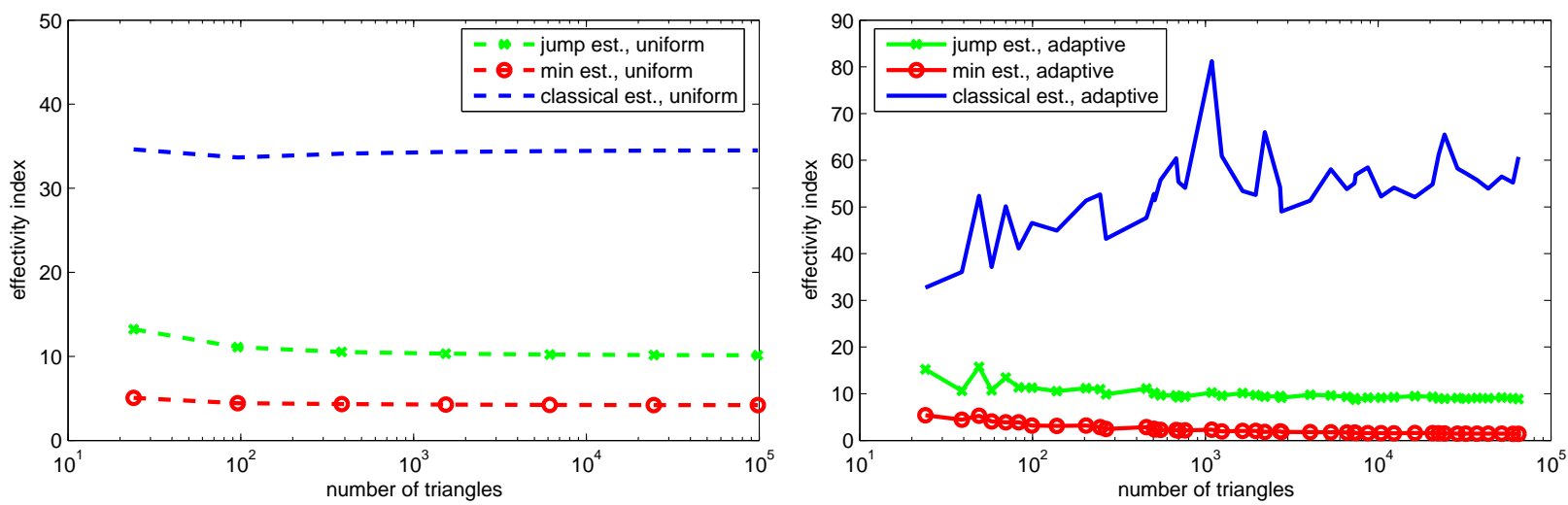

Figure 8. Effectivity indices of the jump estimator (12), the minimization estimator (21), and the classical residual estimator (13) against the number of elements in uniformly (left) and adaptively (right) refined meshes

the number of elements in both uniformly and adaptively refined meshes. As the solution posses a singularity here, the adaptive approach leads to an important improvement of the error attained for a given number of unknowns. The effectivity indices are then shown in the right part of Figure 7 . We can see that for uniform refinement, we overestimate by a factor of approximately 4.2, whereas for adaptive refinement, this value is close to 1.3. It thus seems that there is no asymptotic exactness in this case, but still the overestimation factor is very mild. As for the first test case, the minimization estimator represents an important improvement over the classical residual estimator (13), see Figure 8. The effectivity index of the latter one is around 34.5 for uniform refinement and around 65 for adaptive refinement. From this figure, we can also see that the original jump estimator (12) performs much better than the classical residual one but not as well as the minimization one, with the effectivity indices equal to, respectively, 10 and 8.75. It is very interesting to compare the diffusive flux and residual parts of the jump and minimization estimators in this case, as we do it in Figure 9. It again turns out that in the jump estimator, the major part of the estimation is represented by the residual estimator and only a minor one by the diffusive flux one. Performing the local minimization of Section 3 in this case only equilibrates the residual and diffusive flux estimators on uniformly refined meshes. On adaptively refined meshes, however, as in the previous case, the residual estimator gets superconvergent and consequently insignificant on fine meshes. In particular, the improvements in the effectivity index using the local minimization are much more significant here, compare Figures 4 and 8.

We have paid in this case a particular attention to adaptive mesh refinement using our error estimators. As in the previous case, an element was marked for refinement if the estimator exceeded $25 \%$ of the maximal element estimators and the adaptive mesh refinement possibilities of FreeFem $++[9]$ were exploited. A particular remark here goes to the fact that on each refinement step, FreeFem ++ generates a completely new mesh on the basis of the given error distribution and this new mesh is thus not a simple refinement of the previous one. Another point is that our numerical experiments suggest that one gets better results (better error decreasing with the number of elements) in adaptive refinement when the elements for refinement are chosen using the jump estimator and not the minimization one; this seems to be in agreement with the theoretical developments, since we prove the local efficiency for the jump estimator, see Theorem 1.3. We thus for the best results use the jump estimator distribution in order to choose elements for adaptive refinement and the minimization estimator in order to control the overall error. To conclude, the predicted and actual error distribution as given by the jump estimator in the uniform and adaptive cases are plotted in Figures 10 and 11, respectively; we can see that they match excellently. 

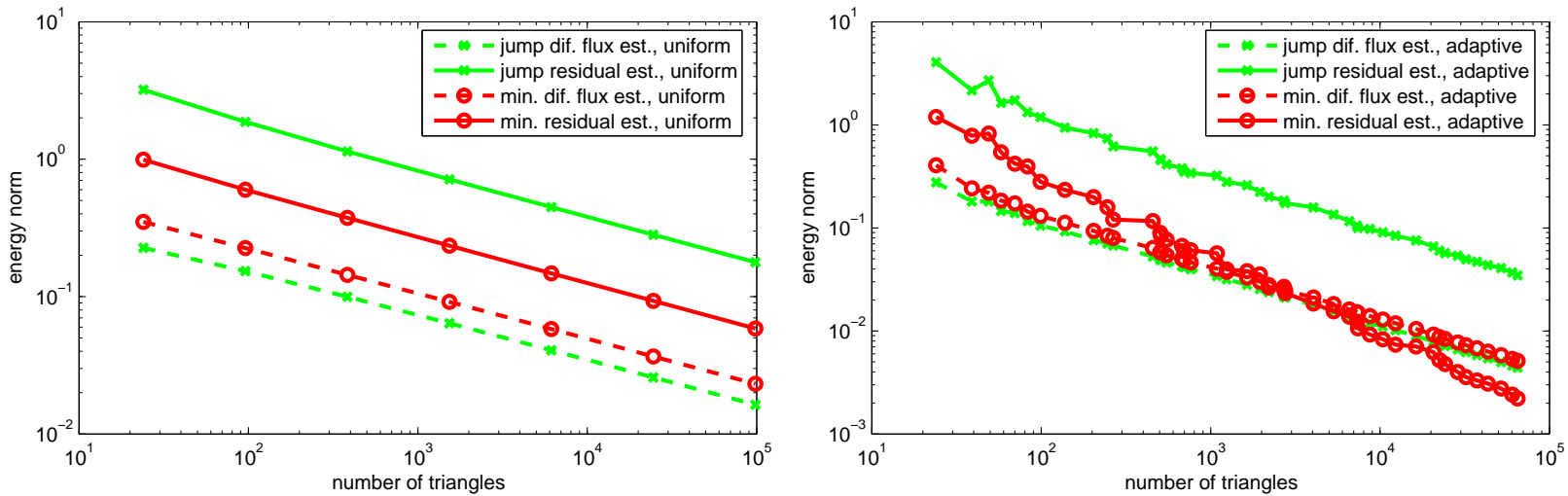

Figure 9. Comparison of the jump estimator (12) and of the minimization estimator (21) components against the number of elements in uniformly (left) and adaptively (right) refined meshes
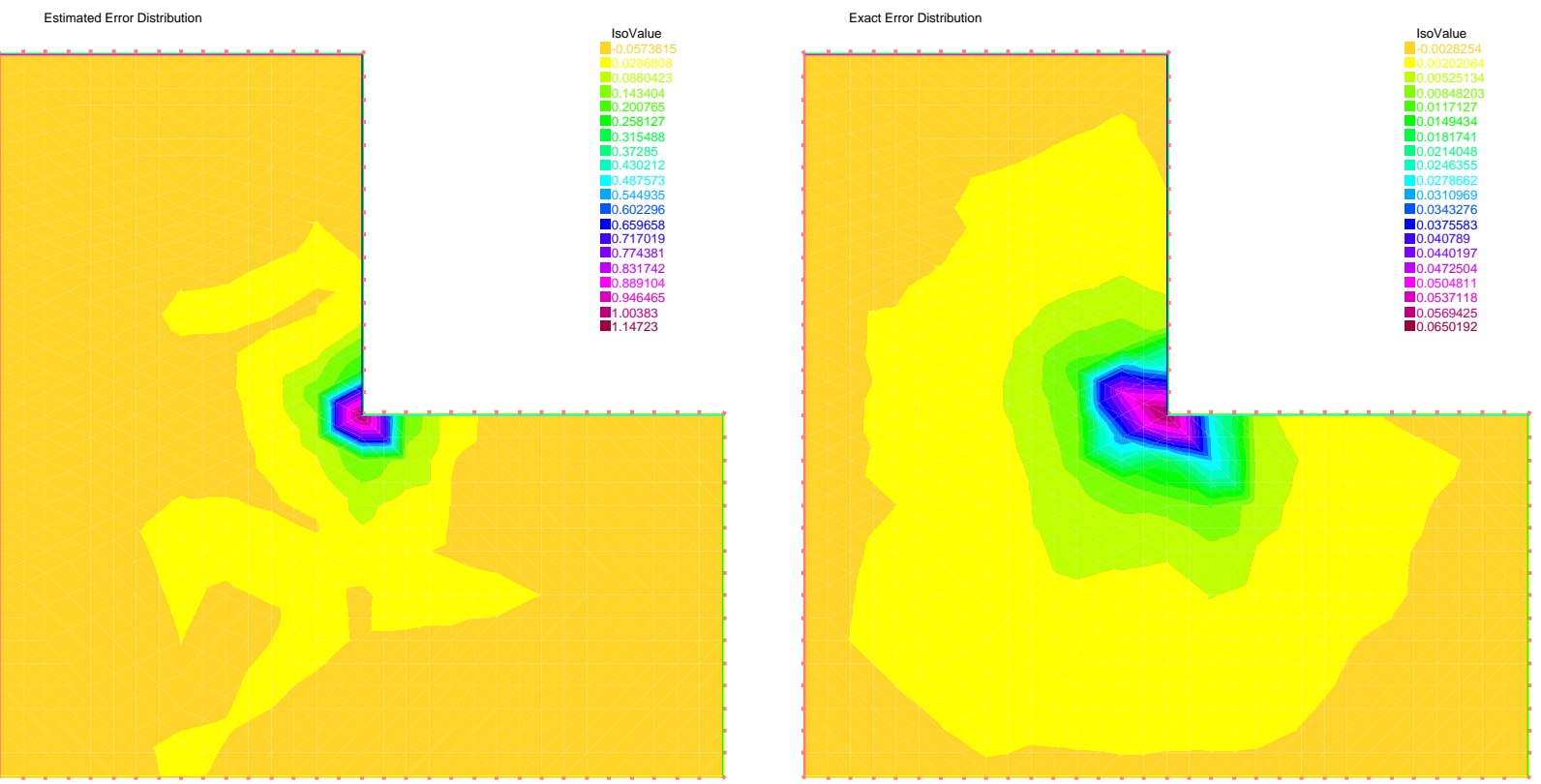

FiguRE 10. Estimated error (left) and exact error (right) distribution using jump estimator and uniform refinement

\section{REFERENCES}

[1] Ainsworth, M., and Oden, J. T. A posteriori error estimation in finite element analysis. Pure and Applied Mathematics (New York). Wiley-Interscience [John Wiley \& Sons], New York, 2000.

[2] Bank, R. E., And Rose, D. J. Some error estimates for the box method. SIAM J. Numer. Anal. 24, 4 (1987), $777-787$.

[3] Bebendorf, M. A note on the Poincaré inequality for convex domains. Z. Anal. Anwendungen 22, 4 (2003), 751-756.

[4] Carstensen, C., and Funken, S. A. Constants in Clément-interpolation error and residual based a posteriori error estimates in finite element methods. East-West J. Numer. Math. 8, 3 (2000), 153-175.

[5] Cheddadi, I., Fučí́, R., Prieto, M. I., and Vohralík, M. Guaranteed and robust a posteriori error estimates for singularly perturbed reaction-diffusion problems. Preprint R08018, Laboratoire Jacques-Louis Lions, submitted for publication, 2008. 

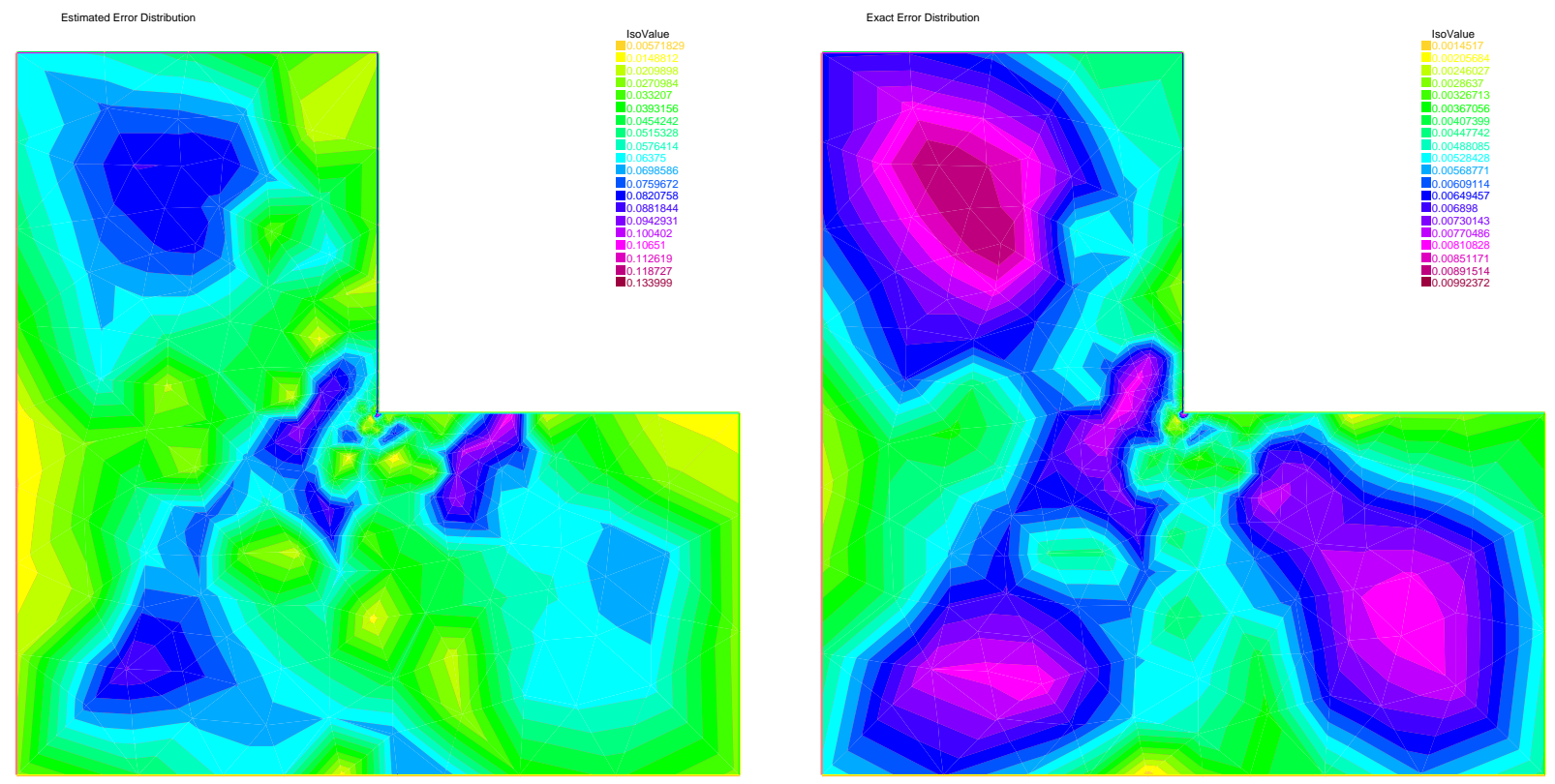

FiguRE 11. Estimated error (left) and exact error (right) distribution using jump estimator and adaptive refinement

[6] Destuynder, P., And Métivet, B. Explicit error bounds in a conforming finite element method. Math. Comp. 68, 228 (1999), $1379-1396$.

[7] Ern, A., Stephansen, A. F., and Vohralík, M. Guaranteed and robust a posteriori error estimation based on flux reconstruction for discontinuous Galerkin methods. Preprint R07050, Laboratoire Jacques-Louis Lions, submitted for publication, 2007.

[8] Eymard, R., Gallouët, T., and Herbin, R. Finite volume methods. In Handbook of Numerical Analysis, Vol. VII. NorthHolland, Amsterdam, 2000, pp. 713-1020.

[9] Hecht, F., Pironneau, O., Le Hyaric, A., and Ohtsuka, K. FreeFem++. Tech. rep., Laboratoire Jacques-Louis Lions, Université Pierre et Marie Curie, Paris, http://www.freefem.org/ff++, 2007.

[10] Hughes, T. J. R., Engel, G., Mazzei, L., and Larson, M. G. The continuous Galerkin method is locally conservative. J. Comput. Phys. 163, 2 (2000), 467-488.

[11] Jiránek, P., Strakoš, Z., And Vohralík, M. A posteriori error estimates including algebraic error: computable upper bounds and stopping criteria for iterative solvers, submitted for publication, 2008.

[12] Konotov, S. Two-sided a posteriori error estimates for linear elliptic problems with mixed boundary conditions. Appl. Math. 52, 3 (2007), 235-249.

[13] Ladevèze, P., and Leguillon, D. Error estimate procedure in the finite element method and applications. SIAM J. Numer. Anal. 20, 3 (1983), 485-509.

[14] Luce, R., and Wohlmuth, B. I. A local a posteriori error estimator based on equilibrated fluxes. SIAM J. Numer. Anal. 42, 4 (2004), 1394-1414.

[15] Payne, L. E., and Weinberger, H. F. An optimal Poincaré inequality for convex domains. Arch. Rational Mech. Anal. 5 (1960), 286-292 (1960).

[16] Repin, S., and Sauter, S. Functional a posteriori estimates for the reaction-diffusion problem. C. R. Math. Acad. Sci. Paris 343, 5 (2006), 349-354.

[17] Strouboulis, T., BabušKa, I., and Gangaraj, S. K. Guaranteed computable bounds for the exact error in the finite element solution. II. Bounds for the energy norm of the error in two dimensions. Internat. J. Numer. Methods Engrg. 47, 1-3 (2000), 427-475. Richard H. Gallagher Memorial Issue.

[18] VejchodskÝ, T. Guaranteed and locally computable a posteriori error estimate. IMA J. Numer. Anal. 26, 3 (2006), 525-540.

[19] Verfürth, R. A review of a posteriori error estimation and adaptive mesh-refinement techniques. Teubner-Wiley, Stuttgart, 1996. 
[20] Vohralík, M. On the discrete Poincaré-Friedrichs inequalities for nonconforming approximations of the Sobolev space $H^{1}$. Numer. Funct. Anal. Optim. 26, 7-8 (2005), 925-952.

[21] Vohralík, M. Residual flux-based a posteriori error estimates for finite volume and related locally conservative methods. Numer. Math., DOI 10.1007/s00211-008-0168-4, 2008.

[22] Vohralík, M. A posteriori error estimates for lowest-order mixed finite element discretizations of convection-diffusion-reaction equations. SIAM J. Numer. Anal. 45, 4 (2007), 1570-1599.

[23] Vohralík, M. Guaranteed and fully robust a posteriori error estimates for conforming discretizations of diffusion problems with discontinuous coefficients. Preprint R08009, Laboratoire Jacques-Louis Lions, submitted for publication, 2008.

[24] Vohralík, M. A posteriori error estimation in the conforming finite element method based on its local conservativity and using local minimization. C. R. Math. Acad. Sci. Paris. 346, 11-12 (2008), 687-690.

[25] Zienkiewicz, O. C., ANd Zhu, J. Z. A simple error estimator and adaptive procedure for practical engineering analysis. Internat. J. Numer. Methods Engrg. 24, 2 (1987), 337-357. 